\title{
A preconditioned fast finite volume scheme for a fractional differential equation discretized on a locally refined composite mesh
}

\author{
Jinhong Jia ${ }^{a}$ Hong Wang ${ }^{b}$ \\ ${ }^{a}$ School of Mathematics, Shandong University, Jinan, Shandong 250100, China \\ ${ }^{b}$ Department of Mathematics, University of South Carolina, Columbia, South Carolina \\ 29208, USA (e-mail address: hwang@math.sc.edu, telephone: 803-777-4321. fax: \\ 803-777-6527)
}

\begin{abstract}
Numerical methods for fractional differential equations generate full stiffness matrices, which were traditionally solved via Gaussian type direct solvers that require $O\left(N^{3}\right)$ of computational work and $O\left(N^{2}\right)$ of memory to store where $N$ is the number of spatial grid points in the discretization. We develop a preconditioned fast Krylov subspace iterative method for the efficient and faithful solution of finite volume schemes defined on a locally refined composite mesh for fractional differential equations to resolve boundary layers of the solutions. Numerical results are presented to show the utility of the method.
\end{abstract}

Keywords: circulant matrix, fast solution method, finite volume method, fractional differential equation, locally refined mesh, Toeplitz matrix

\section{Introduction}

Fractional differential equations were shown to provide an adequate and accurate description of transport processes that exhibit anomalous diffusive behavior, which cannot be modeled properly by canonical second-order diffusion equations $[2,15]$. Extensive research has been conducted in the development of numerical methods for fractional differential equations $[5,7,9,10,12$, $13,14,18,26,27,28]$. Because of the nonlocal nature of fractional differential operators, numerical methods for space-fractional differential equations usually generate full stiffness matrices and were traditionally solved via Gaussian 
elimination. This requires $O\left(N^{3}\right)$ of computational work per time step and $O\left(N^{2}\right)$ of memory to store, where $N$ is the number of spatial grid points in the discretization, and represents a significant increase over the computational cost and memory requirement of numerical methods of second-order diffusion equations.

We proved that the stiffness matrices of finite difference schemes for spacefractional differential equations can be decomposed as a sum of diagonalmultiply-Toeplitz matrices (or their block analogue in multidimensional cases), and consequently developed fast solution methods that has a computational work account of $O(N \log N)$ per iteration or time step and has a memory requirement of $O(N)$ via the fast Fourier transform (FFT), while retaining the accuracy of the underlying numerical schemes [19, 21, 23]. A fast finite volume scheme was also developed in [20]. Numerical experiments showed the significant reduction in computational cost and memory requirement over the traditional methods. As they were based upon FFT, these fast methods were limited to uniform spatial meshes.

It was not realized until recently $[11,24,25]$ that solutions to fractional differential equations may exhibit boundary layer and poor regularity even if the diffusivity coefficient and right-hand side are smooth. For example, it is easy to check that

$$
u(x)=x^{1-\beta}, \quad x \in(0,1),
$$

with $0<\beta<1$, is the solution to the boundary-value problem of the homogeneous fractional differential equation with a constant diffusivity coefficient

$$
\begin{aligned}
D\left({ }_{0} D_{x}^{-\beta} D u\right) & =0, \quad x \in(0,1), \\
u(0)=0, \quad u(1) & =1
\end{aligned}
$$

where $D$ represents the first-order differential operator, and ${ }_{0} D_{x}^{-\beta}$ and ${ }_{x} D_{1}^{-\beta}$ represent the left and right fractional integral operators [16]

$$
\left\{\begin{aligned}
{ }_{0} D_{x}^{-\beta} g(x) & :=\frac{1}{\Gamma(\beta)} \int_{0}^{x}(x-s)^{\beta-1} g(s) d s, \\
{ }_{x} D_{1}^{-\beta} g(x) & :=\frac{1}{\Gamma(\beta)} \int_{x}^{1}(s-x)^{\beta-1} g(s) d s
\end{aligned}\right.
$$

Where $\Gamma(\cdot)$ is a Gamma function.

It is clear that the solution $u$ has a boundary layer. It was proved in [25] that the true solution $u$ is not in the fractional Sobolev space $H^{\frac{3}{2}-\beta}(0,1)$, 
but is still in the Besov space $B_{\infty}^{\frac{3}{2}-\beta}\left(L^{2}(0,1)\right)[1]$. From a numerical point of view, a numerical scheme with a uniform mesh is probably not anticipated to efficiently resolve the boundary layer of the solution $u$ at $x=0$, and so a numerical scheme that is discretized on a locally refined composite mesh is desired. This, in turn, implies that fractional finite difference methods are out of the question, as they are based on the discretization of the GrünwaldLetnikov fractional derivatives that are inherently defined on uniform meshes $[16]$.

A fundamental approach to resolve fronts in solutions to differential equations is to employ an adaptive mechanism. However, in the context of fractional differential equations, any local change in a mesh will destroy the global structure of the stiffness matrix. Hence, one should take great care in the development of an adaptive method by delicately balancing the adaptivity of the method and the structure of the adapted meshes. As a preliminary first step towards the eventual resolution of the steep fronts present in the solutions to fractional differential equations, the goal of this paper is to derive a fast and faithful finite volume scheme on a locally refined composite mesh for the inhomogeneous Dirichlet boundary-value problem of the conservative variable-coefficient Caputo fractional differential equation of order $2-\beta$ with $0<\beta<1[2,6,7]$

$$
\begin{gathered}
-D\left(K(x)\left(\gamma_{0} D_{x}^{-\beta}+(1-\gamma)_{x} D_{1}^{-\beta}\right) D u\right)=f(x), \quad 0<x<1, \\
u(0)=u_{l}, \quad u(1)=u_{r} .
\end{gathered}
$$

Here $K(x)$ is the diffusivity coefficient, $0 \leq \gamma \leq 1$ indicates the relative weight of forward versus backward transition probability, $f(x)$ is the source and sink term, and $u_{l}$ and $u_{r}$ are the prescribed Dirichlet boundary data. The rest of the paper is organized as follows. In section 2 we derive a finite volume scheme on a composite mesh. In section 3 we study the structure of the stiffness matrix. In section 4 we develop a fast and faithful Krylov subspace method for the finite volume scheme. In section 5 we present an efficient preconditioner. In section 6 we carry out numerical experiments to investigate the performance of the fast method. In section 7 we discuss extensions and future directions.

\section{A finite volume scheme on a composite mesh}

For simplicity of presentation, we assume that the boundary layer of the true solution is located at the left endpoint $x=0$. We will consider the 
general case that the boundary layers of the true solution appear at both endpoints $x=0$ and $x=1$ at the end of the paper. To resolve the potential boundary layer of the true solution while maximizing the efficiency of the derived numerical scheme, we introduce a composite mesh as follows:

We begin by a uniform partition of mesh size $h:=1 / n$ for a positive integer $n$. Then we introduce a geometrically decreased mesh on the subinterval $[0, h]$ starting from $x=h$ successfully for $m$ times with $m$ being a positive number. Let $N:=m+n$. We follow the convention to label the nodes in the composite mesh from left to right as follows: we let $h_{1}:=2^{-m} h$ be the finest mesh, $h_{i}:=2^{-(m-(i-2))} h$ for $i=2, \cdots, m+1$ be the mesh sizes of a geometrically increasing mesh, and $h_{i}:=h$ for $i=m+2, \cdots, N$ be the uniformly coarse mesh size where. Then we set $x_{0}:=0$ and $x_{i}:=x_{i-1}+h_{i}$ for $i=1,2, \cdots, N$ to be the sequence of space nodes of the composite mesh. It is clear that $x_{m+1}=h$ and $x_{N}=1$.

Let $\left\{\phi_{i}\right\}_{i=0}^{N}$ be the set of hat functions such that $\phi_{i}\left(x_{i}\right)=1$ and $\phi_{i}\left(x_{j}\right)=0$ for $j \neq i$. The finite volume approximation $u_{h}$ to the true solution $u$ of problem (4) can be expressed as

$$
u_{h}(x):=\sum_{j=1}^{N-1} u_{j} \phi_{j}(x)+u_{l} \phi_{0}(x)+u_{r} \phi_{N}(x) .
$$

Let $x_{i-\frac{1}{2}}:=\left(x_{i-1}+x_{i}\right) / 2$ to be the midpoints of the interval $\left[x_{i-1}, x_{i}\right]$ for $i=1,2, \ldots, N$. We integrate the governing equation in (4) on $\left[x_{i-\frac{1}{2}}, x_{i+\frac{1}{2}}\right]$ for $i=1,2, \cdots, N-1$ to obtain the following finite volume scheme

$$
\begin{aligned}
- & \sum_{j=1}^{N-1} u_{j} \int_{x_{i-\frac{1}{2}}}^{x_{i+\frac{1}{2}}} D\left(K(x)\left(\gamma_{0} D_{x}^{-\beta}+(1-\gamma)_{x} D_{1}^{-\beta}\right) D \phi_{j}(x)\right) d x \\
& =\int_{x_{i-\frac{1}{2}}}^{x_{i+\frac{1}{2}}} f(x) d x \\
& -\int_{x_{i-\frac{1}{2}}}^{x_{i+\frac{1}{2}}} D\left[K(x)\left(\gamma_{0} D_{x}^{-\beta}+(1-\gamma)_{x} D_{1}^{-\beta}\right) D\left(u_{l} \phi_{0}(x)+u_{r} \phi_{N}(x)\right)\right] d x .
\end{aligned}
$$

Thus, the finite volume scheme can be written in a matrix form

$$
A u=f
$$

where $u:=\left[u_{1}, u_{2}, \cdots, u_{N-1}\right]^{T}, f:=\left[f_{1}, f_{2}, \cdots, f_{N-1}\right]^{T}$, and $A:=\left[a_{i, j}\right]_{i, j=1}^{N-1}$ 
with

$$
\begin{aligned}
a_{i, j}= & \left.\left(K(x)\left(\gamma_{0} D_{x}^{-\beta}+(1-\gamma)_{x} D_{1}^{-\beta}\right) D \phi_{j}(x)\right)\right|_{x=x_{i-\frac{1}{2}}} \\
& \left.-\left(K(x) \gamma_{0} D_{x}^{-\beta}+(1-\gamma)_{x} D_{1}^{-\beta}\right) D \phi_{j}(x)\right)\left.\right|_{x=x_{i+\frac{1}{2}}} \\
f_{i}= & \int_{x_{i-\frac{1}{2}}}^{x_{i+\frac{1}{2}}} f(x) d x \\
+ & \left.K(x)\left(\gamma_{0} D_{x}^{-\beta}+(1-\gamma)_{x} D_{1}^{-\beta}\right) D\left(u_{l} \phi_{0}(x)+u_{r} \phi_{N}(x)\right)\right|_{x=x_{i+\frac{1}{2}}} \\
- & \left.K(x)\left(\gamma_{0} D_{x}^{-\beta}+(1-\gamma)_{x} D_{1}^{-\beta}\right) D\left(u_{l} \phi_{0}(x)+u_{r} \phi_{N}(x)\right)\right|_{x=x_{i-\frac{1}{2}}} .
\end{aligned}
$$

\section{Structure of the stiffness matrix}

The finite volume scheme (5) looks like the one for the canonical secondorder diffusion equation, but has fundamental difference. Although the hat functions $\phi_{j}$ have local support, (3) reveals that ${ }_{0} D_{x}^{-\beta} D \phi_{j}$ and ${ }_{x} D_{1}^{-\beta} D \phi_{j}$ have global support. Therefore, the stiffness matrix $A$ is a full matrix, which requires $O\left(N^{2}\right)$ of memory to store. Numerical schemes for space-fractional differential equations were traditionally solved by Gaussian type of direct solvers that requires $O\left(N^{3}\right)$ of computations $[6,7,12,14]$.

\subsection{The entries of the stiffness matrix}

To develop an efficient and faithful method, we have to explore the structure of the stiffness matrix $A$. We go through tedious algebraic manipulations to evaluate all the entires of the stiffness matrix and present them below. For simplicity of presentation, we express the stiffness matrix $A$ as the following $3 \times 3$ block matrix

$$
A=\left[\begin{array}{ccc}
a_{1,1} & A_{1, l} & A_{1, r} \\
A_{l, 1} & A_{l, l} & A_{l, r} \\
A_{r, 1} & A_{r, l} & A_{r, r}
\end{array}\right] .
$$

Here $A_{1, l}=\left[a_{1,2}, \ldots, a_{1, m+1}\right], A_{1, r}=\left[a_{1, m+2}, \ldots, a_{1, N-1}\right], A_{l, 1}=\left[a_{2,1}, \ldots, a_{m+1,1}\right]^{T}$, and $A_{r, 1}=\left[a_{m+2,1}, \ldots, a_{N-1,1}\right]^{T}$; the submatrices $A_{l, l}$ of order $m$ and $A_{r, r}$ of order $n-2$ represent the stiffness matrices corresponding to the nodes $x_{2}, \ldots, x_{m+1}=h$ on the geometrically gridded mesh and the nodes $x_{m+2}, \ldots, x_{N-1}$ on the uniform mesh, respectively. The submatrices $A_{l, r}$ of order $m$-by- $(n-2)$ and $A_{r, l}$ of order $(n-2)$-by- $m$ represent the coupling between the nodes 
$x_{2}, \ldots, x_{m+1}$ in the geometrically gridded mesh and the nodes $x_{m+2}, \ldots, x_{N-1}$ in the uniform mesh, respectively.

First, we have

$$
\begin{aligned}
a_{11} & =\frac{\gamma K_{\frac{1}{2}}}{\Gamma(\beta+1) h_{1}^{1-\beta}} \frac{1}{2^{\beta}}-\frac{(1-\gamma) K_{\frac{1}{2}}}{\Gamma(\beta+1) h_{1}^{1-\beta}}\left[\left(\frac{3}{2}\right)^{\beta}-2\left(\frac{1}{2}\right)^{\beta}\right] \\
& -\frac{\gamma K_{\frac{3}{2}}}{\Gamma(\beta+1) h_{1}^{1-\beta}}\left[\left(\frac{3}{2}\right)^{\beta}-2\left(\frac{1}{2}\right)^{\beta}\right]+\frac{(1-\gamma) K_{\frac{3}{2}}}{\Gamma(\beta+1) h_{1}^{1-\beta}} \frac{1}{2^{\beta}}
\end{aligned}
$$

The entries of $A_{l, 1}$ are given by

$$
\begin{aligned}
a_{21} & =\frac{\gamma K_{\frac{3}{2}}}{\Gamma(\beta+1) h_{1}^{1-\beta}}\left[\left(\frac{3}{2}\right)^{\beta}-2\left(\frac{1}{2}\right)^{\beta}\right]-\frac{(1-\gamma) K_{\frac{3}{2}}}{\Gamma(\beta+1) h_{1}^{1-\beta}} \frac{1}{2^{\beta}} \\
& -\frac{\gamma K_{\frac{5}{2}}}{\Gamma(\beta+1) h_{1}^{1-\beta}}\left[3^{\beta}-2 \cdot 2^{\beta}+1\right]
\end{aligned}
$$

and

$$
\begin{aligned}
a_{i, 1}= & \frac{\gamma K_{i-\frac{1}{2}}}{\Gamma(\beta+1) h_{1}^{1-\beta}}\left[\left(3 \cdot 2^{i-3}\right)^{\beta}-2\left(3 \cdot 2^{i-3}-1\right)^{\beta}+\left(3 \cdot 2^{i-3}-2\right)^{\beta}\right] \\
- & \frac{\gamma K_{i+\frac{1}{2}}}{\Gamma(\beta+1) h_{1}^{1-\beta}}\left[\left(3 \cdot 2^{i-2}\right)^{\beta}-2\left(3 \cdot 2^{i-2}-1\right)^{\beta}+\left(3 \cdot 2^{i-2}-2\right)^{\beta}\right] \\
& \quad 3 \leq i \leq m+1 .
\end{aligned}
$$

The entries of $A_{r, 1}$ are given by

$$
\begin{array}{r}
a_{i, 1}=\frac{\gamma K_{i-\frac{1}{2}}}{\Gamma(\beta+1) h_{1} h^{-\beta}}\left[\left(i-m-\frac{1}{2}\right)^{\beta}-2\left(i-m-\frac{1}{2}-\frac{1}{2^{m}}\right)^{\beta}\right. \\
\left.+\left(i-m-\frac{1}{2}-\frac{1}{2^{m-1}}\right)^{\beta}\right] \\
-\frac{\gamma K_{i+\frac{1}{2}}}{\Gamma(\beta+1) h_{1} h^{-\beta}}\left[\left(i-m+\frac{1}{2}\right)^{\beta}-2\left(i-m+\frac{1}{2}-\frac{1}{2^{m}}\right)^{\beta}\right. \\
\left.+\left(i-m+\frac{1}{2}-\frac{1}{2^{m-1}}\right)^{\beta}\right], \quad m+2 \leq i \leq N-1 .
\end{array}
$$


The entries of $A_{1, l}$ are given by

$$
\begin{aligned}
a_{1,2} & =\frac{(1-\gamma) K_{\frac{3}{2}}}{\Gamma(\beta+1) h_{2}^{1-\beta}}\left[\frac{3}{2}\left(\frac{3}{2}\right)^{\beta}-\left(\frac{1}{2}\right)^{\beta}-\frac{1}{2}\left(\frac{7}{2}\right)^{\beta}\right] \\
& -\frac{\gamma K_{\frac{5}{2}}}{\Gamma(\beta+1) h_{2}^{1-\beta}} \frac{1}{2^{\beta}}-\frac{(1-\gamma) K_{\frac{5}{2}}}{\Gamma(\beta+1) h_{2}^{1-\beta}}\left[\frac{3}{2}\left(\frac{1}{2}\right)^{\beta}-\frac{1}{2}\left(\frac{5}{2}\right)^{\beta}\right]
\end{aligned}
$$

and

$$
\begin{aligned}
a_{1, j} & =\frac{(1-\gamma) K_{\frac{1}{2}}}{\Gamma(\beta+1) h_{j}^{1-\beta}}\left[\frac{3}{2}\left(2-2^{1-j}\right)^{\beta}-\left(1-2^{1-j}\right)^{\beta}\right. \\
& \left.-\frac{1}{2}\left(4-2^{1-j}\right)^{\beta}\right]-\frac{(1-\gamma) K_{\frac{3}{2}}}{\Gamma(\beta+1) h_{j}^{1-\beta}}\left[\frac{3}{2}\left(2-3 \cdot 2^{1-j}\right)^{\beta}\right. \\
& \left.-\left(1-3 \cdot 2^{1-j}\right)^{\beta}-\frac{1}{2}\left(4-3 \cdot 2^{1-j}\right)^{\beta}\right], \quad 3 \leq j \leq m+1 .
\end{aligned}
$$

The entries of $A_{1, r}$ are given by

$$
\begin{aligned}
a_{1, j}= & \frac{(1-\gamma) K_{\frac{1}{2}}}{\Gamma(\beta+1) h^{1-\beta}}\left[2\left(j-m-\frac{1}{2^{m+1}}\right)^{\beta}-\left(j-m-1-\frac{1}{2^{m+1}}\right)^{\beta}\right. \\
& \left.-\left(j-m+1-\frac{1}{2^{m+1}}\right)^{\beta}\right] \\
- & \frac{(1-\gamma) K_{\frac{3}{2}}}{\Gamma(\beta+1) h^{1-\beta}}\left[2\left(j-m-\frac{3}{2^{m+1}}\right)^{\beta}-\left(j-m-1-\frac{3}{2^{m+1}}\right)^{\beta}\right. \\
& \left.-\left(j-m+1-\frac{3}{2^{m+1}}\right)^{\beta}\right], \quad m+2 \leq i \leq N-1 .
\end{aligned}
$$

We now turn to the sub-matrix $A_{l, l}$. Its diagonal entries are given by

$$
\begin{aligned}
a_{i, i} & =\frac{\gamma K_{i-\frac{1}{2}}}{\Gamma(\beta+1) h_{i}^{1-\beta}} \frac{1}{2^{\beta}}-\frac{(1-\gamma) K_{i-\frac{1}{2}}}{\Gamma(\beta+1) h_{i}^{1-\beta}}\left[\frac{1}{2}\left(\frac{5}{2}\right)^{\beta}-\frac{3}{2}\right] \\
& -\frac{\gamma K_{i+\frac{1}{2}}}{\Gamma(\beta+1) h_{i}^{1-\beta}}\left[2^{\beta}-\frac{3}{2}\right]+\frac{(1-\gamma) K_{i+\frac{1}{2}}}{\Gamma(\beta+1) h_{i}^{1-\beta}} \frac{1}{2}-, \quad 2 \leq i \leq m+1 .
\end{aligned}
$$

The sub-triangular entries of $A_{l, l}$ are given by

$$
\begin{aligned}
a_{i, i-1} & =\frac{\gamma K_{i-\frac{1}{2}}}{\Gamma(\beta+1) h_{i-1}^{1-\beta}}\left[2^{\beta}-\frac{3}{2}\right]-\frac{(1-\gamma) K_{i-\frac{1}{2}}}{\Gamma(\beta+1) h_{i-1}^{1-\beta}} \frac{1}{2} \\
& -\frac{\gamma K_{i+\frac{1}{2}}}{\Gamma(\beta+1) h_{i-1}^{1-\beta}}\left[5^{\beta}-\frac{3}{2} \cdot 4^{\beta}+\frac{1}{2} \cdot 2^{\beta}\right], \quad 3 \leq i \leq m+1
\end{aligned}
$$


and

$$
\begin{aligned}
a_{i, j}= & \frac{\gamma K_{i-\frac{1}{2}}}{\Gamma(\beta+1) h_{j}^{1-\beta}}\left[\left(3 \cdot 2^{i-j-1}-1\right)^{\beta}-\frac{3}{2}\left(3 \cdot 2^{i-j-1}-2\right)^{\beta}\right. \\
& \left.+\frac{1}{2}\left(3 \cdot 2^{i-j-1}-4\right)^{\beta}\right]-\frac{\gamma K_{i+\frac{1}{2}}}{\Gamma(\beta+1) h_{j}^{1-\beta}}\left[\left(3 \cdot 2^{i-j}-1\right)^{\beta}\right. \\
& \left.-\frac{3}{2}\left(3 \cdot 2^{i-j}-2\right)^{\beta}+\frac{1}{2}\left(3 \cdot 2^{i-j}-4\right)^{\beta}\right], \\
& 4 \leq i \leq m+1,1 \leq j \leq i-2 .
\end{aligned}
$$

Similarly, the super-triangular entries of $A_{l, l}$ are given by

$$
\begin{aligned}
& a_{i, i+1}=\frac{(1-\gamma) K_{i-\frac{1}{2}}}{\Gamma(\beta+1) h_{i+1}^{1-\beta}}\left[\frac{3}{2}\left(\frac{5}{4}\right)^{\beta}-\left(\frac{1}{4}\right)^{\beta}-\frac{1}{2}\left(\frac{13}{4}\right)^{\beta}\right] \\
&-\left.\frac{\gamma K_{i+\frac{1}{2}}}{\Gamma(\beta+1) h_{i+1}^{1-\beta}} \frac{1}{2^{\beta}}-\frac{(1-\gamma) K_{i+\frac{1}{2}}}{\Gamma(\beta+1) h_{i+1}^{1-\beta}}\left[\frac{3}{2}\left(\frac{1}{2}\right)^{\beta}-\frac{1}{2}\left(\frac{5}{2}\right)^{\beta}\right)\right], \\
& 2 \leq i \leq m
\end{aligned}
$$

and

$$
\begin{aligned}
a_{i, j}= & \frac{(1-\gamma) K_{i-\frac{1}{2}}}{\Gamma(\beta+1) h_{j}^{1-\beta}}\left[\frac{3}{2}\left(2-3 \cdot 2^{i-j-1}\right)^{\beta}-\left(1-3 \cdot 2^{i-j-1}\right)^{\beta}\right. \\
& \left.-\frac{1}{2}\left(4-3 \cdot 2^{i-j-1}\right)^{\beta}\right]-\frac{(1-\gamma) K_{i+\frac{1}{2}}}{\Gamma(\beta+1) h_{j}^{1-\beta}}\left[\frac{3}{2}\left(2-3 \cdot 2^{i-j}\right)^{\beta}\right. \\
& \left.-\left(1-3 \cdot 2^{i-j}\right)^{\beta}-\frac{1}{2}\left(4-3 \cdot 2^{i-j}\right)^{\beta}\right], \\
& \quad 2 \leq i \leq m-1, i+2 \leq j \leq m+1 .
\end{aligned}
$$


The entries of $A_{r, l}$ are given by

$$
\begin{aligned}
& a_{i, j}= \frac{\gamma K_{i-\frac{1}{2}}}{\Gamma(\beta+1) h_{j}^{1-\beta}}\left[\left(2^{m-j+2}\left(i-m-\frac{1}{2}\right)-1\right)^{\beta}\right. \\
&\left.-\frac{3}{2}\left(2^{m-j+2}\left(i-m-\frac{1}{2}\right)-2\right)^{\beta}+\frac{1}{2}\left(2^{m-j+2}\left(i-m-\frac{1}{2}\right)-4\right)^{\beta}\right] \\
&- \frac{\gamma K_{i+\frac{1}{2}}}{\Gamma(\beta+1) h_{j}^{1-\beta}}\left[\left(2^{m-j+2}\left(i-m+\frac{1}{2}\right)-1\right)^{\beta}\right. \\
&\left.-\frac{3}{2}\left(2^{m-j+2}\left(i-m+\frac{1}{2}\right)-2\right)^{\beta}+\frac{1}{2}\left(2^{m-j+2}\left(i-m+\frac{1}{2}\right)-4\right)^{\beta}\right], \\
& m+2 \leq i \leq N-1,2 \leq j \leq m+1, j \neq i-1 .
\end{aligned}
$$

Here $j=i-1$ occurs only when $i=m+2$ and $j=m+1$. That is, $a_{m+2, m+1}$ is the only entry in $A_{r, l}$ that is not of the form in (20) but is given by the following expression

$$
\begin{aligned}
a_{m+2, m+1} & =\frac{\gamma K_{m+\frac{3}{2}}}{\Gamma(\beta+1) h_{m+1}^{1-\beta}}\left[2^{\beta}-\frac{3}{2}\right]-\frac{(1-\gamma) K_{m+\frac{3}{2}}}{\Gamma(\beta+1) h_{m+1}^{1-\beta}} \frac{1}{2} \\
& -\frac{\gamma K_{m+\frac{5}{2}}}{\Gamma(\beta+1) h_{m+1}^{1-\beta}}\left[5^{\beta}-\frac{3}{2} \cdot 4^{\beta}+\frac{1}{2} \cdot 2^{\beta}\right] .
\end{aligned}
$$

Similarly, the entries of $A_{l, r}$ are given by

$$
\begin{gathered}
a_{i, j}=\frac{(1-\gamma) K_{i-\frac{1}{2}}}{\Gamma(\beta+1) h^{1-\beta}}\left[2\left((j-m)-3 \cdot 2^{i-m-3}\right)^{\beta}-\left((j-m-1)-3 \cdot 2^{i-m-3}\right)^{\beta}\right. \\
\left.-\left((j-m+1)-3 \cdot 2^{i-m-3}\right)^{\beta}\right] \\
-\frac{(1-\gamma) K_{i+\frac{1}{2}}}{\Gamma(\beta+1) h^{1-\beta}}\left[2\left((j-m)-3 \cdot 2^{i-m-2}\right)^{\beta}-\left((j-m-1)-3 \cdot 2^{i-m-2}\right)^{\beta}\right. \\
\left.-\left((j-m+1)-3 \cdot 2^{i-m-2}\right)^{\beta}\right] \\
2 \leq i \leq m+1, m+2 \leq j \leq N-2, j \neq i+1 .
\end{gathered}
$$

We notice that in the preceding expressions, $j=i+1$ occurs only when $i=m+1$ and $j=m+2$. That is, the entry $a_{m+1, m+2}$ is the only entry in 
$A_{l, r}$ that cannot expressed as (22). Its expression is given below

$$
\begin{aligned}
a_{m+1, m+2} & =\frac{(1-\gamma) K_{m+\frac{1}{2}}}{\Gamma(\beta+1) h_{m+2}^{1-\beta}}\left[\frac{3}{2}\left(\frac{5}{4}\right)^{\beta}-\left(\frac{1}{4}\right)^{\beta}-\frac{1}{2}\left(\frac{13}{4}\right)^{\beta}\right] \\
& -\frac{\gamma K_{m+\frac{3}{2}}}{\Gamma(\beta+1) h_{m+2}^{1-\beta}} \frac{1}{2^{\beta}}-\frac{(1-\gamma) K_{m+\frac{3}{2}}}{\Gamma(\beta+1) h_{m+2}^{1-\beta}}\left[\frac{3}{2}\left(\frac{1}{2}\right)^{\beta}-\frac{1}{2}\left(\frac{5}{2}\right)^{\beta}\right] .
\end{aligned}
$$

Finally, we turn the sub-matrix $A_{r, r}$. Its diagonal entries are given by

$$
\begin{gathered}
a_{i, i}=\frac{\gamma K_{i-\frac{1}{2}}}{\Gamma(\beta+1) h^{1-\beta}} \frac{1}{2^{\beta}}-\frac{(1-\gamma) K_{i-\frac{1}{2}}}{\Gamma(\beta+1) h^{1-\beta}}\left[\left(\frac{3}{2}\right)^{\beta}-2\left(\frac{1}{2}\right)^{\beta}\right] \\
-\frac{\gamma K_{i+\frac{1}{2}}}{\Gamma(\beta+1) h^{1-\beta}}\left[\left(\frac{3}{2}\right)^{\beta}-2\left(\frac{1}{2}\right)^{\beta}\right]+\frac{(1-\gamma) K_{i+\frac{1}{2}}}{\Gamma(\beta+1) h^{1-\beta}} \frac{1}{2^{\beta}} \\
m+2 \leq i \leq N-1 .
\end{gathered}
$$

The sub-triangular entries of $A_{r, r}$ are given by

$$
\begin{aligned}
& a_{i, i-1}= \frac{\gamma K_{i-\frac{1}{2}}}{\Gamma(\beta+1) h^{1-\beta}}\left[\left(\frac{3}{2}\right)^{\beta}-2\left(\frac{1}{2}\right)^{\beta}\right]-\frac{(1-\gamma) K_{i-\frac{1}{2}}}{\Gamma(\beta+1) h^{1-\beta}} \frac{1}{2^{\beta}} \\
&- \frac{\gamma K_{i+\frac{1}{2}}}{\Gamma(\beta+1) h^{1-\beta}}\left[\left(\frac{5}{2}\right)^{\beta}-2\left(\frac{3}{2}\right)^{\beta}+\left(\frac{1}{2}\right)^{\beta}\right] \\
& m+3 \leq i \leq N-1
\end{aligned}
$$

and

$$
\begin{gathered}
a_{i, j}=\frac{\gamma K_{i-\frac{1}{2}}}{\Gamma(\beta+1) h^{1-\beta}}\left[\left(i-j+\frac{1}{2}\right)^{\beta}-2\left(i-j-\frac{1}{2}\right)^{\beta}+\left(i-j-\frac{3}{2}\right)^{\beta}\right] \\
-\frac{\gamma K_{i+\frac{1}{2}}}{\Gamma(\beta+1) h^{1-\beta}}\left[\left(i-j+\frac{3}{2}\right)^{\beta}-2\left(i-j+\frac{1}{2}\right)^{\beta}+\left(i-j-\frac{1}{2}\right)^{\beta}\right] \\
m+4 \leq i \leq N-1, \quad m+2 \leq j \leq i-2 .
\end{gathered}
$$

The super-triangular entries of $A_{r, r}$ are given by

$$
\begin{aligned}
& a_{i, i+1}= \frac{(1-\gamma) K_{i-\frac{1}{2}}}{\Gamma(\beta+1) h^{1-\beta}}\left[2\left(\frac{3}{2}\right)^{\beta}-\left(\frac{1}{2}\right)^{\beta}-\left(\frac{5}{2}\right)^{\beta}\right] \\
&- \frac{\gamma K_{i+\frac{1}{2}}}{\Gamma(\beta+1) h^{1-\beta}} \frac{1}{2^{\beta}}-\frac{(1-\gamma) K_{i+\frac{1}{2}}}{\Gamma(\beta+1) h^{1-\beta}}\left[2\left(\frac{1}{2}\right)^{\beta}-\left(\frac{3}{2}\right)^{\beta}\right], \\
& m+2 \leq i \leq N-2
\end{aligned}
$$


and

$$
\begin{gathered}
a_{i, j}=\frac{(1-\gamma) K_{i-\frac{1}{2}}}{\Gamma(\beta+1) h^{1-\beta}}\left[2\left(j-i+\frac{1}{2}\right)^{\beta}-\left(j-i-\frac{1}{2}\right)^{\beta}-\left(j-i+\frac{3}{2}\right)^{\beta}\right] \\
-\frac{(1-\gamma) K_{i+\frac{1}{2}}}{\Gamma(\beta+1) h^{1-\beta}}\left[2\left(j-i-\frac{1}{2}\right)^{\beta}-\left(j-i-\frac{3}{2}\right)^{\beta}-\left(j-i+\frac{1}{2}\right)^{\beta}\right] \\
m+2 \leq j \leq N-3, j+2 \leq i \leq N-1 .
\end{gathered}
$$

\subsection{Structure of the stiffness matrix}

In this subsection we study the structure of the stiffness matrix $A$.

Theorem 1. The submatrices $A_{l, l}$ and $A_{r, r}$ can be decomposed as

$$
\begin{aligned}
A_{l, l}=\frac{1}{\Gamma(\beta+1)} & {\left[\operatorname{diag}\left(K_{l}^{-}\right)\left(\gamma Q_{l}+(1-\gamma) Q_{r}\right)\right.} \\
& \left.-\operatorname{diag}\left(K_{l}^{+}\right)\left(\gamma P_{l}+(1-\gamma) P_{r}\right)\right] \operatorname{diag}\left(\left\{h_{i}^{\beta-1}\right\}_{i=1}^{m}\right), \\
A_{r, r}=\frac{h^{\beta-1}}{\Gamma(\beta+1)} & {\left[\operatorname{diag}\left(K_{r}^{-}\right)\left(\gamma S+(1-\gamma) R^{T}\right)\right.} \\
& \left.-\operatorname{diag}\left(K_{r}^{+}\right)\left(\gamma R+(1-\gamma) S^{T}\right)\right]
\end{aligned}
$$

where the vectors $K_{l}^{+}, K_{l}^{-}, K_{r}^{+}$, and $K_{r}^{-}$are defined by

$$
\begin{array}{ll}
K_{l}^{+}=\left[K_{\frac{5}{2}}, K_{\frac{7}{2}}, \cdots, K_{m+\frac{3}{2}}\right], & K_{r}^{+}=\left[K_{m+\frac{5}{2}}, K_{m+\frac{7}{2}}, \cdots, K_{m+n-\frac{1}{2}}\right], \\
K_{l}^{-}=\left[K_{\frac{3}{2}}, K_{\frac{5}{2}}, \cdots, K_{m+\frac{1}{2}}\right], & K_{r}^{-}=\left[K_{m+\frac{3}{2}}, K_{m+\frac{5}{2}}, \cdots, K_{m+n-\frac{3}{2}}\right]
\end{array}
$$

and the matrices $P_{l}, P_{r}, Q_{l}, Q_{r}, R$, and $S$ are defined by

$$
P_{l}:=\left[\begin{array}{cccccc}
p_{0}^{(l, \beta)} & p_{-1}^{(l, \beta)} & 0 & \ldots & \ldots & 0 \\
p_{1}^{(l, \beta)} & p_{0}^{(l, \beta)} & p_{-1}^{(l, \beta)} & 0 & \ldots & 0 \\
p_{2}^{(l, \beta)} & p_{1}^{(l, \beta)} & p_{0}^{(l, \beta)} & p_{l,-1}^{(\beta)} & 0 & 0 \\
\vdots & \ddots & \ddots & \ddots & \ddots & \vdots \\
\vdots & \ddots & \ddots & p_{1}^{(l, \beta)} & p_{0}^{(l, \beta)} & p_{-1}^{(l, \beta)} \\
p_{m-1}^{(l, \beta)} & p_{m-2}^{(l, \beta)} & \cdots & \cdots & p_{1}^{(l, \beta)} & p_{0}^{(l, \beta)}
\end{array}\right],
$$




$$
\begin{aligned}
& P_{r}:=\left[\begin{array}{cccccc}
p_{0}^{(r, \beta)} & p_{-1}^{(r, \beta)} & p_{-2}^{(r, \beta)} & \ldots & \ldots & p_{1-m}^{(r, \beta)} \\
0 & p_{0}^{(r, \beta)} & p_{-1}^{(r, \beta)} & p_{-2}^{(r, \beta)} & \ldots & p_{2-m}^{(r, \beta)} \\
0 & 0 & p_{0}^{(r, \beta)} & p_{-1}^{(r, \beta)} & \ldots & p_{3-m}^{(r, \beta)} \\
\vdots & \ddots & \ddots & \ddots & \ddots & \vdots \\
0 & \ddots & \ddots & 0 & p_{0}^{(r, \beta)} & p_{-1}^{(r, \beta)} \\
0 & \cdots & \cdots & 0 & 0 & p_{0}^{(r, \beta)}
\end{array}\right], \\
& Q_{l}:=\left[\begin{array}{cccccc}
q_{0}^{(l, \beta)} & 0 & 0 & \cdots & \ldots & 0 \\
q_{1}^{(l, \beta)} & q_{0}^{(l, \beta)} & 0 & 0 & \ldots & 0 \\
q_{2}^{(l, \beta)} & q_{1}^{(l \beta)} & q_{0}^{(l, \beta)} & 0 & \ldots & 0 \\
\vdots & \ddots & \ddots & \ddots & \ddots & \vdots \\
\vdots & \ddots & \ddots & q_{1}^{(\beta)} & q_{0}^{(l, \beta)} & 0 \\
q_{m-1}^{(l, \beta)} & q_{m-2}^{(l, \beta)} & \cdots & \cdots & q_{1}^{(l, \beta)} & q_{0}^{(l, \beta)}
\end{array}\right], \\
& Q_{r}:=\left[\begin{array}{cccccc}
q_{0}^{(r, \beta)} & q_{-1}^{(r, \beta)} & q_{-2}^{(r, \beta)} & \ldots & \ldots & q_{1-m}^{(r, \beta)} \\
q_{1}^{(r, \beta)} & q_{0}^{(r, \beta)} & q_{-1}^{(r, \beta)} & q_{-2}^{(r, \beta)} & \ldots & q_{2-m}^{(r, \beta)} \\
0 & q_{1}^{(r, \beta)} & q_{0}^{(r, \beta)} & q_{-1}^{(r, \beta)} & \ldots & q_{3-m}^{(r, \beta)} \\
\vdots & \ddots & \ddots & \ddots & \ddots & \vdots \\
0 & \ddots & \ddots & q_{1}^{(r, \beta)} & q_{0}^{(r, \beta)} & q_{-1}^{(r, \beta)} \\
0 & \ldots & \ldots & 0 & q_{1}^{(r, \beta)} & q_{0}^{(r, \beta)}
\end{array}\right],
\end{aligned}
$$




$$
R:=\left[\begin{array}{cccccc}
r_{0}^{(\beta)} & r_{-1}^{(\beta)} & 0 & \cdots & 0 & 0 \\
r_{1}^{(\beta)} & r_{0}^{(\beta)} & r_{-1}^{(\beta)} & 0 & \cdots & 0 \\
r_{2}^{(\beta)} & r_{1}^{(\beta)} & r_{0}^{(\beta)} & r_{-1}^{(\beta)} & 0 & \vdots \\
\vdots & \ddots & \ddots & \ddots & \ddots & \vdots \\
\vdots & \ddots & \ddots & r_{1}^{(\beta)} & r_{0}^{(\beta)} & r_{-1}^{(\beta)} \\
r_{n-3}^{(\beta)} & r_{n-4}^{(\beta)} & \cdots & \cdots & r_{1}^{(\beta)} & r_{0}^{(\beta)}
\end{array}\right]
$$

and

$$
S:=\left[\begin{array}{cccccc}
s_{0}^{(\beta)} & 0 & 0 & \cdots & \cdots & 0 \\
s_{1}^{(\beta)} & s_{0}^{(\beta)} & 0 & 0 & \cdots & 0 \\
s_{2}^{(\beta)} & s_{1}^{(\beta)} & s_{0}^{(\beta)} & 0 & \cdots & 0 \\
\vdots & \ddots & \ddots & \ddots & \ddots & \vdots \\
\vdots & \ddots & \ddots & s_{1}^{(\beta)} & s_{0}^{(\beta)} & 0 \\
s_{n-3}^{(\beta)} & s_{n-4}^{(\beta)} & \cdots & \cdots & s_{1}^{(\beta)} & s_{0}^{(\beta)}
\end{array}\right] .
$$

Here the entries of matrices $P_{l}, P_{r}, Q_{l}, Q_{r}, R$, and $S$ are given by

$$
\begin{gathered}
p_{i}^{(l, \beta)}= \begin{cases}\left(\frac{1}{2}\right)^{\beta}, & i=1, \\
2^{\beta}-\frac{3}{2}, & i=0, \\
\left(3 \cdot 2^{i}-1\right)^{\beta}-\frac{3}{2}\left(3 \cdot 2^{i}-2\right)^{\beta}+\frac{1}{2}\left(3 \cdot 2^{i}-4\right)^{\beta}, & 1 \leq i \leq m,\end{cases} \\
p_{i}^{(r, \beta)}= \begin{cases}-\frac{1}{2}, & i=0, \\
\frac{3}{2}\left(\frac{1}{2}\right)^{\beta}-\frac{1}{2}\left(\frac{5}{2}\right)^{\beta}, & i=-1, \\
\frac{3}{2}\left(2-3 \cdot 2^{i}\right)^{\beta}-\left(1-3 \cdot 2^{i}\right)^{\beta}-\frac{1}{2}\left(4-3 \cdot 2^{i}\right)^{\beta}, & 1-m \leq i \leq-2,\end{cases}
\end{gathered}
$$




$$
\begin{aligned}
& q_{i}^{(l, \beta)}= \begin{cases}\left(\frac{1}{2}\right)^{\beta}, & i=0, \\
2^{\beta}-\frac{3}{2}, & i=1, \\
\left(3 \cdot 2^{i-1}-1\right)^{\beta}-\frac{3}{2}\left(3 \cdot 2^{i-1}-2\right)^{\beta}+\frac{1}{2}\left(3 \cdot 2^{i-1}-4\right)^{\beta}, & 2 \leq i \leq m-1,\end{cases} \\
& q_{i}^{(r, \beta)}= \begin{cases}-\frac{1}{2}, & i=1, \\
\frac{3}{2}-\frac{1}{2}\left(\frac{5}{2}\right)^{\beta}, & i=0, \\
\frac{3}{2}\left(2-3 \cdot 2^{i-1}\right)^{\beta}-\left(1-3 \cdot 2^{i-1}\right)^{\beta}-\frac{1}{2}\left(4-3 \cdot 2^{i-1}\right)^{\beta}, & 1-m \leq i \leq-1,\end{cases} \\
& r_{i}^{(\beta)}= \begin{cases}\left(\frac{1}{2}\right)^{\beta}, & i=-1, \\
\left(\frac{3}{2}\right)^{\beta}-2\left(\frac{1}{2}\right)^{\beta}, & i=0, \\
\left(i+\frac{3}{2}\right)^{\beta}-2\left(i+\frac{1}{2}\right)^{\beta}+\left(i-\frac{1}{2}\right)^{\beta}, & 1 \leq i \leq n-3\end{cases}
\end{aligned}
$$

and

$$
s_{i}^{(\beta)}= \begin{cases}\left(\frac{1}{2}\right)^{\beta}, & i=0, \\ \left(\frac{3}{2}\right)^{\beta}-2\left(\frac{1}{2}\right)^{\beta}, & i=1, \\ \left(i+\frac{1}{2}\right)^{\beta}-2\left(i-\frac{1}{2}\right)^{\beta}+\left(i-\frac{3}{2}\right)^{\beta}, & 2 \leq i \leq n-3 .\end{cases}
$$

Proof. The second decomposition in equation (29) can be obtained by a direction examination of equations (24)-(28), while the first decomposition can be obtained in a similar way by a direction examination of equations (15)-(19).

Theorem 2. The submatrices $A_{l, r}$ and $A_{r, l}$ can be decomposed as

$$
\begin{aligned}
& A_{l, r}=\frac{(1-\gamma) h^{\beta-1}}{\Gamma(\beta+1)}\left(\operatorname{diag}\left(K_{l}^{-}\right) E-\operatorname{diag}\left(K_{l}^{+}\right) D\right), \\
& A_{r, l}=\frac{\gamma}{\Gamma(\beta+1)}\left(\operatorname{diag}\left(K_{r}^{-}\right) H-\operatorname{diag}\left(K_{r}^{+}\right) G\right) \operatorname{diag}\left(\left\{h_{i}^{\beta-1}\right\}_{i=1}^{m}\right)
\end{aligned}
$$


where the matrices $D=\left[d_{i, j}\right]_{i, j=1}^{m, n-2}, E=\left[e_{i, j}\right]_{i, j=1}^{m, n-2}, G=\left[g_{i, j}\right]_{i, j=1}^{n-2, m}$, and $H=\left[h_{i, j}\right]_{i, j=1}^{n-2, m}$ are defined by

$$
\begin{aligned}
d_{i, j} & =2\left(j+1-3 \cdot 2^{i-m-1}\right)^{\beta}-\left(j-3 \cdot 2^{i-m-1}\right)^{\beta} \\
& -\left(j+2-3 \cdot 2^{i-m-1}\right)^{\beta}, \quad(i, j) \neq(m, 1), \\
d_{m, 1} & =\frac{\gamma}{1-\gamma}\left(\frac{1}{2}\right)^{\beta}+\frac{3}{2}\left(\frac{1}{2}\right)^{\beta}-\frac{1}{2}\left(\frac{5}{2}\right)^{\beta}, \\
e_{i, j} \quad & =2\left(j+1-3 \cdot 2^{i-m-2}\right)^{\beta}-\left(j-3 \cdot 2^{i-m-2}\right)^{\beta} \\
& -\left(j+2-3 \cdot 2^{i-m-2}\right)^{\beta}, \\
g_{i, j} & =\left[2^{m-j+1}\left(i+\frac{3}{2}\right)-1\right]^{\beta}-\frac{3}{2}\left[2^{m-j+1}\left(i+\frac{3}{2}\right)-2\right]^{\beta} \\
& +\frac{1}{2}\left[2^{m-j+1}\left(i+\frac{3}{2}\right)-4\right]^{\beta}, \\
& =\left[2^{m-j+1}\left(i+\frac{1}{2}\right)-1\right]^{\beta}-\frac{3}{2}\left[2^{m-j+1}\left(i+\frac{1}{2}\right)-2\right]^{\beta} \\
h_{i, j} & +\frac{1}{2}\left[2^{m-j+1}\left(i+\frac{1}{2}\right)-4\right]^{\beta}, \quad(i, j) \neq(1, m) \\
h_{1, m} & =2^{\beta}-\frac{1}{2}-\frac{1}{2 \gamma} .
\end{aligned}
$$

Proof. The first decomposition in equations (30) can be verified directly from equations (22) and (23), while the second decomposition in (30) can be checked from equations (20) and (21).

\section{A fast Krylov subspace method with efficient storage}

Equipped with Theorems 1 and 2 about the structure of the stiffness matrix $A$, we are now in a position to develop fast solution method with an efficient storage mechanism.

Theorem 3. The stiffness matrix A can be stored in $O(m N)$ memory. For each vector $w \in \mathbb{R}^{N}$, Aw can be evaluated in $O((m+\log N) N)$ operations.

Proof. From the matrix decomposition (7), we observe that the first row and column of $A$ have $2 N-1$ parameters to be stored. Next, Theorem 1 shows 
that $K_{l}^{+}, K_{l}^{-}, K_{r}^{+}$, and $K_{r}^{-}$have $N$ parameters to store, $P_{l}, P_{r}, Q_{l}$, and $Q_{r}$ have $(4 m+2)$ parameters to store, $R$ and $S$ have $(2 n-3)$ parameters to store, and the diagonal matrix consisting of $h_{i}$ has $m$ parameters to store. In short, $A_{l, l}$ and $A_{r, r}$ require $O(N)$ of memory to store. Finally, Theorem 2 shows that the matrices $D, E, G$, and $H$, and so $A_{l, r}$ and $A_{r, l}$ require $O(m(N-m))$ parameters to store.

It is known that an $n \times n$ Toeplitz matrix $T$ of the form

$$
T=\left[\begin{array}{ccccc}
t_{0} & t_{-1} & \cdots & t_{2-n} & t_{1-n} \\
t_{1} & t_{0} & t_{-1} & \cdots & t_{2-n} \\
\vdots & t_{1} & t_{0} & \ddots & \vdots \\
t_{n-2} & \cdots & \ddots & \ddots & t_{-1} \\
t_{n-1} & t_{n-2} & \cdots & t_{1} & t_{0}
\end{array}\right]
$$

can be embedded into a $2 n \times 2 n$ circulant matrix $C_{2 n}$ as follows

$$
C_{2 n}=\left[\begin{array}{cc}
T & T^{\prime} \\
T^{\prime} & T
\end{array}\right] \text { with } T^{\prime}=\left[\begin{array}{ccccc}
0 & t_{n-1} & \cdots & t_{2} & t_{1} \\
t_{1-n} & 0 & \ddots & \ddots & t_{2} \\
\vdots & \ddots & \ddots & \ddots & \vdots \\
t_{-2} & \ddots & \ddots & 0 & t_{n-1} \\
t_{-1} & t_{-2} & \cdots & t_{1-n} & 0
\end{array}\right] .
$$

A circulant matrix $C_{2 n}$ can be diagonalized by the Fourier transform matrix $F_{2 n}$

$$
C_{2 n}=F_{2 n}^{-1} \operatorname{diag}\left(F_{2 n} c\right) F_{2 n}
$$

where $c$ is the first column vector of $C_{2 n}$. It is well known that the matrixvector multiplication $F_{2 n} w_{2 n}$ for any $w_{2 n} \in \mathbb{R}^{2 n}$ can be carried out in $O(2 n \log (2 n))=$ $O(n \log (n))$ operations by the discrete fast Fourier transform. Thus, $C_{2 n} w_{2 n}$ can be evaluated in $O(n \log (n))$ operations. For any $w_{n} \in \mathbb{R}^{n}$, by choosing $w_{2 n}:=\left[w_{n}^{T}, 0^{T}\right]^{T}, T_{n} w_{n}$ can be evaluated in $O(n \log n)$ operations. Thus, Theorem 1 ensures that $A_{l, l} w_{m}$ and $A_{r, r} W_{n-1}$ can be evaluated in $O(m \log m$ and $O(n \log n)$ operations, respectively. 
However, the full matrices $D, E, G$, and $H$ are not Toeplitz, and the corresponding matrix-vector multiplications can be carried out in $O(m n)=$ $O(m N)$ operations. Hence, Theorem 2 concludes that the corresponding matrix-vector multiplication to $A_{l, r}$ and $A_{r, l}$ can be evaluated in $O(m N)$ operations.

By exploiting the structure of the stiffness matrix $A$, Theorem 3 reduces the computational cost of $O\left(N^{3}\right)$ by Gaussian elimination to $O((m+\log N) N)$ per iteration by any Krylov subspace method, and the memory requirement from $O\left(N^{2}\right)$ of a straightforward storage mechanism to $O(m N)$. Hence, Theorem 3 presents a substantial improvement both in computational cost and in memory requirement.

However, both the improved computational cost and memory requirement still depends on the number of points $m$ in the locally refined region. The counterexample in section 1 shows that fractional differential equation might have a boundary layer of the form $x^{1-\beta}$ even for smooth diffusivity coefficient and right-hand side. Computationally, $m$ number of points may be placed in the boundary layer cell. Sometimes $m=O(N)$ number of points may be used in the first cell to resolve a boundary layer, as the Shishikin mesh $[8,17]$ that was used to resolve exponential boundary layers present in canonical second-order advection-diffusion equations. In an extreme scenario like this, Theorem 3 does not suffice. A second look at the proof of Theorem 3 reveals that the storage of and a fast matrix-vector multiplication by $A_{l, r}$ and $A_{r, l}$ dominate the memory requirement and computational cost. This motivates the following theorem.

Theorem 4. The submatrices $A_{l, r}$ and $A_{r, l}$ can be approximately stored in $O(N)$ memory. The matrix-vector multiplications can be approximately evaluated in $O(N \log N)$ operations.

Proof. We begin with the matrix $A_{l, r}$, which is equivalent to looking at $D$ and $E$ in equations (31). We notice that $D$ is not a Toeplitz matrix. Nevertheless, the entries of $D$ exhibit a clear pattern. For $1 \leq i \leq m$ and $1 \leq j \leq n-2$, we apply the binomial expansion to expand the three terms of $d_{i, j}$ at $j+1$ 
to obtain

$$
\begin{aligned}
2(j+ & \left.1-3 \cdot 2^{i-m-1}\right)^{\beta} \\
= & 2(j+1)^{\beta}\left(1-\frac{3 \cdot 2^{i-m-1}}{j+1}\right)^{\beta} \\
= & 2(j+1)^{\beta}\left[1-\beta \frac{3 \cdot 2^{i-m-1}}{j+1}+\left(\begin{array}{l}
\beta \\
2
\end{array}\right)\left(\frac{3 \cdot 2^{i-m-1}}{j+1}\right)^{2}\right. \\
& \left.\quad-\left(\begin{array}{c}
\beta \\
3
\end{array}\right)\left(\frac{3 \cdot 2^{i-m-1}}{j+1}\right)^{3}+\left(\begin{array}{c}
\beta \\
4
\end{array}\right)\left(\frac{3 \cdot 2^{i-m-1}}{j+1}\right)^{4}+O\left(\frac{1}{(j+1)^{5}}\right)\right], \\
\left(j-3 \cdot 2^{i-m-1}\right)^{\beta} & (j+1)^{\beta}\left(1-\frac{1+3 \cdot 2^{i-m-1}}{j+1}\right)^{\beta} \\
= & (j+1)^{\beta}\left[1-\beta\left(\frac{1+3 \cdot 2^{i-m-1}}{j+1}\right)+\left(\begin{array}{l}
\beta \\
2
\end{array}\right)\left(\frac{1+3 \cdot 2^{i-m-1}}{j+1}\right)^{2}\right. \\
& \left.-\left(\begin{array}{c}
\beta \\
3
\end{array}\right)\left(\frac{1+3 \cdot 2^{i-m-1}}{j+1}\right)^{3}+\left(\begin{array}{c}
\beta \\
4
\end{array}\right)\left(\frac{1+3 \cdot 2^{i-m-1}}{j+1}\right)^{4}+O\left(\frac{1}{(j+1)^{5}}\right)\right],
\end{aligned}
$$

and

$$
\begin{aligned}
(j+ & \left.2-3 \cdot 2^{i-m-1}\right)^{\beta} \\
= & (j+1)^{\beta}\left(1+\frac{1-3 \cdot 2^{i-m-1}}{j+1}\right)^{\beta} \\
= & (j+1)^{\beta}\left[1+\beta\left(\frac{1-3 \cdot 2^{i-m-1}}{j+1}\right)+\left(\begin{array}{c}
\beta \\
2
\end{array}\right)\left(\frac{1-3 \cdot 2^{i-m-1}}{j+1}\right)^{2}\right. \\
& \left.+\left(\begin{array}{l}
\beta \\
3
\end{array}\right)\left(\frac{1-3 \cdot 2^{i-m-1}}{j+1}\right)^{3}+\left(\begin{array}{l}
\beta \\
4
\end{array}\right)\left(\frac{1-3 \cdot 2^{i-m-1}}{j+1}\right)^{4}+O\left(\frac{1}{(j+1)^{5}}\right)\right] .
\end{aligned}
$$

We combine the preceding three expansions to obtain the following expansion 
for $d_{i, j}$ with $(i, j) \neq(m, 1)$

$$
\begin{aligned}
d_{i, j}= & 2\left(j+1-3 \cdot 2^{i-m-1}\right)^{\beta}-\left(j-3 \cdot 2^{i-m-1}\right)^{\beta} \\
& -\left(j+2-3 \cdot 2^{i-m-1}\right)^{\beta} \\
= & -(j+1)^{\beta}\left[2\left(\begin{array}{l}
\beta \\
2
\end{array}\right) \frac{1}{(j+1)^{2}}-6\left(\begin{array}{c}
\beta \\
3
\end{array}\right) \frac{3 \cdot 2^{i-m-1}}{(j+1)^{3}}\right. \\
& \left.+\left(\begin{array}{c}
\beta \\
4
\end{array}\right)\left(\frac{12\left(3 \cdot 2^{i-m-1}\right)^{2}}{(j+1)^{4}}+\frac{2}{(j+1)^{4}}\right)+O\left(\frac{1}{(j+1)^{5}}\right)\right] \\
= & -2\left(\begin{array}{l}
\beta \\
2
\end{array}\right) \frac{1}{(j+1)^{2-\beta}}-2\left(\begin{array}{c}
\beta \\
4
\end{array}\right) \frac{1}{(j+1)^{4-\beta}}+18\left(\begin{array}{c}
\beta \\
3
\end{array}\right) \frac{2^{i-m-1}}{(j+1)^{3-\beta}} \\
& -108\left(\begin{array}{l}
\beta \\
4
\end{array}\right) \frac{2^{2(i-m-1)}}{(j+1)^{4-\beta}}+O\left(\frac{1}{(j+1)^{5-\beta}}\right) .
\end{aligned}
$$

Thus, if we drop the local truncation error term, we obtain the following low-rank decomposition for the matrix $D$

$$
\begin{aligned}
D \approx & -2\left(\begin{array}{l}
\beta \\
2
\end{array}\right)[1,1, \ldots, 1]^{T}\left[\frac{1}{2^{2-\beta}}, \frac{1}{3^{2-\beta}}, \ldots, \frac{1}{(n-1)^{2-\beta}}\right] \\
& -2\left(\begin{array}{l}
\beta \\
4
\end{array}\right)[1,1, \ldots, 1]^{T}\left[\frac{1}{2^{4-\beta}}, \frac{1}{3^{4-\beta}}, \ldots, \frac{1}{(n-1)^{4-\beta}}\right] \\
& +18\left(\begin{array}{l}
\beta \\
3
\end{array}\right)\left[2^{-m}, 2^{-m+1}, \ldots, 2^{-1}\right]^{T}\left[\frac{1}{2^{3-\beta}}, \frac{1}{3^{3-\beta}}, \ldots, \frac{1}{(n-1)^{3-\beta}}\right] \\
& -108\left(\begin{array}{l}
\beta \\
4
\end{array}\right)\left[2^{-2 m}, 2^{-2 m+2}, \ldots, 2^{-2}\right]^{T}\left[\frac{1}{2^{4-\beta}}, \frac{1}{3^{4-\beta}}, \ldots, \frac{1}{(n-1)^{4-\beta}}\right]
\end{aligned}
$$

where all the row vectors are $m$-dimensional vectors and the column vectors are $(n-2)$-dimensional vectors. If we replace the component $i-m-1$ in (33) by $i-m-2$, we obtain the following expansion for the matrix $E$

$$
\begin{aligned}
E \approx & -2\left(\begin{array}{l}
\beta \\
2
\end{array}\right)[1,1, \ldots, 1]^{T}\left[\frac{1}{2^{2-\beta}}, \frac{1}{3^{2-\beta}}, \ldots, \frac{1}{(n-1)^{2-\beta}}\right] \\
& -2\left(\begin{array}{l}
\beta \\
4
\end{array}\right)[1,1, \ldots, 1]^{T}\left[\frac{1}{2^{4-\beta}}, \frac{1}{3^{4-\beta}}, \ldots, \frac{1}{(n-1)^{4-\beta}}\right] \\
& +18\left(\begin{array}{l}
\beta \\
3
\end{array}\right)\left[2^{-m-1}, 2^{-m}, \ldots, 2^{-2}\right]^{T}\left[\frac{1}{2^{3-\beta}}, \frac{1}{3^{3-\beta}}, \ldots, \frac{1}{(n-1)^{3-\beta}}\right] \\
& -108\left(\begin{array}{l}
\beta \\
4
\end{array}\right)\left[2^{-2 m-2}, 2^{-2 m}, \ldots, 2^{-4}\right]^{T}\left[\frac{1}{2^{4-\beta}}, \frac{1}{3^{4-\beta}}, \ldots, \frac{1}{(n-1)^{4-\beta}}\right] .
\end{aligned}
$$


We observe from the decomposition (30) that the matrix $A_{l, r}$ can be approximately stored in $O(N)$ memories and the action of $A_{l, r}$ on any vector can be evaluated in $O(N)$ operations.

We now turn to matrix $G$ and $H$, and so $A_{r, l}$. For $g_{i, j}$ given in (31) with $1 \leq i \leq n-2$ and $1 \leq j \leq m$, we have

$$
\begin{aligned}
\left(2^{m-j+1}\left(i+\frac{3}{2}\right)-1\right)^{\beta} & \\
= & \left(2^{m+j-1}\left(i+\frac{3}{2}\right)\right)^{\beta}\left(1-2^{j-m-1}\left(i+\frac{3}{2}\right)^{-1}\right)^{\beta} \\
= & \left(2^{m+j-1}\left(i+\frac{3}{2}\right)\right)^{\beta}\left[1-\beta \cdot 2^{j-m-1}\left(i+\frac{3}{2}\right)^{-1}+\left(\begin{array}{c}
\beta \\
2
\end{array}\right)\left(2^{j-m-1}\left(i+\frac{3}{2}\right)^{-1}\right)^{2}\right. \\
& -\left(\begin{array}{c}
\beta \\
3
\end{array}\right)\left(2^{j-m-1}\left(i+\frac{3}{2}\right)^{-1}\right)^{3}+\left(\begin{array}{c}
\beta \\
4
\end{array}\right)\left(2^{j-m-1}\left(i+\frac{3}{2}\right)^{-1}\right)^{4} \\
& \left.+O\left(\left(2^{j-m-1}\left(i+\frac{3}{2}\right)^{-1}\right)^{5}\right)\right], \\
\left(2^{m-}\right. & \left.j+1\left(i+\frac{3}{2}\right)-2\right)^{\beta} \\
= & \left(2^{m+j-1}\left(i+\frac{3}{2}\right)\right)^{\beta}\left(1-2 \cdot 2^{j-m-1}\left(i+\frac{3}{2}\right)^{-1}\right)^{\beta} \\
= & \left(2^{m+j-1}\left(i+\frac{3}{2}\right)\right)^{\beta}\left[1-2 \beta \cdot 2^{j-m-1}\left(i+\frac{3}{2}\right)^{-1}+2^{2}\left(\begin{array}{l}
\beta \\
2
\end{array}\right)\left(2^{j-m-1}\left(i+\frac{3}{2}\right)^{-1}\right)^{2}\right. \\
& -2^{3}\left(\begin{array}{l}
\beta \\
3
\end{array}\right)\left(2^{j-m-1}\left(i+\frac{3}{2}\right)^{-1}\right)^{3}+2^{4}\left(\begin{array}{l}
\beta \\
4
\end{array}\right)\left(2^{j-m-1}\left(i+\frac{3}{2}\right)^{-1}\right)^{4} \\
& \left.+O\left(\left(2^{j-m-1}\left(i+\frac{3}{2}\right)^{-1}\right)^{5}\right)\right],
\end{aligned}
$$


and

$$
\begin{aligned}
& \left(2^{m-j+1}\left(i+\frac{3}{2}\right)-4\right)^{\beta} \\
& =\left(2^{m+j-1}\left(i+\frac{3}{2}\right)\right)^{\beta}\left(1-4 \cdot 2^{j-m-1}\left(i+\frac{3}{2}\right)^{-1}\right)^{\beta} \\
& =\left(2^{m+j-1}\left(i+\frac{3}{2}\right)\right)^{\beta}\left[1-4 \beta \cdot 2^{j-m-1}\left(i+\frac{3}{2}\right)^{-1}+4^{2}\left(\begin{array}{c}
\beta \\
2
\end{array}\right)\left(2^{j-m-1}\left(i+\frac{3}{2}\right)^{-1}\right)^{2}\right. \\
& \quad-4^{3}\left(\begin{array}{l}
\beta \\
3
\end{array}\right)\left(2^{j-m-1}\left(i+\frac{3}{2}\right)^{-1}\right)^{3}+4^{4}\left(\begin{array}{c}
\beta \\
4
\end{array}\right)\left(2^{j-m-1}\left(i+\frac{3}{2}\right)^{-1}\right)^{4} \\
& \left.\quad+O\left(\left(2^{j-m-1}\left(i+\frac{3}{2}\right)^{-1}\right)^{5}\right)\right]
\end{aligned}
$$

We combine the previous three expansions to obtain the following expansion for $g_{i, j}$ with $(i, j) \neq(1, m)$

$$
\begin{aligned}
g_{i, j} & =\left(2^{m-j+1}\left(i+\frac{3}{2}\right)-1\right)^{\beta}-\frac{3}{2}\left(2^{m-j+1}\left(i+\frac{3}{2}\right)-2\right)^{\beta} \\
& +\frac{1}{2}\left(2^{m-j+1}\left(i+\frac{3}{2}\right)-4\right)^{\beta} \\
& \left(2^{m-j+1}\left(i+\frac{3}{2}\right)\right)^{\beta}\left[3\left(\begin{array}{c}
\beta \\
2
\end{array}\right)\left(2^{j-m-1}\left(i+\frac{3}{2}\right)^{-1}\right)^{2}\right. \\
& +21\left(\begin{array}{c}
\beta \\
3
\end{array}\right)\left(2^{j-m-1}\left(i+\frac{3}{2}\right)^{-1}\right)^{3}-105\left(\begin{array}{c}
\beta \\
4
\end{array}\right)\left(2^{j-m-1}\left(i+\frac{3}{2}\right)^{-1}\right)^{4} \\
& \left.+O\left(\left(2^{j-m-1}\left(i+\frac{3}{2}\right)^{-1}\right)^{5}\right)\right] \\
& =3\left(\begin{array}{c}
\beta \\
2
\end{array}\right)\left(2^{j-m-1}\right)^{2-\beta}\left(i+\frac{3}{2}\right)^{\beta-2}+21\left(\begin{array}{c}
\beta \\
3
\end{array}\right)\left(2^{j-m-1}\right)^{3-\beta}\left(i+\frac{3}{2}\right)^{\beta-3} \\
& -105\left(\begin{array}{c}
\beta \\
4
\end{array}\right)\left(2^{j-m-1}\right)^{4-\beta}\left(i+\frac{3}{2}\right)^{\beta-4}+O\left(\left(2^{j-m-1}\left(i+\frac{3}{2}\right)^{-1}\right)^{5-\beta}\right) .
\end{aligned}
$$

Dropping the local truncation error term yields the following low-rank de- 
composition for the matrix $G$

$$
\begin{aligned}
G \approx & 3\left(\begin{array}{l}
\beta \\
2
\end{array}\right)\left[\left(\frac{2}{5}\right)^{2-\beta},\left(\frac{2}{7}\right)^{2-\beta}, \ldots,\left(\frac{2}{2 n-1}\right)^{2-\beta}\right]^{T} \\
& {\left[2^{-m(2-\beta)}, 2^{(1-m)(2-\beta)}, \ldots, 2^{-(2-\beta)}\right] } \\
+ & 21\left(\begin{array}{l}
\beta \\
3
\end{array}\right)\left[\left(\frac{2}{5}\right)^{3-\beta},\left(\frac{2}{7}\right)^{3-\beta}, \ldots,\left(\frac{2}{2 n-1}\right)^{3-\beta}\right]^{T} \\
& {\left[2^{-m(3-\beta)}, 2^{(1-m)(3-\beta)}, \ldots, 2^{-(3-\beta)}\right] } \\
- & 105\left(\begin{array}{c}
\beta \\
4
\end{array}\right)\left[\left(\frac{2}{5}\right)^{4-\beta},\left(\frac{2}{7}\right)^{4-\beta}, \ldots,\left(\frac{2}{2 n-1}\right)^{4-\beta}\right]^{T} \\
& {\left[2^{-m(4-\beta)}, 2^{(1-m)(4-\beta)}, \cdots, 2^{-(4-\beta)}\right] . }
\end{aligned}
$$

If we replace $(i+3 / 2)$ by $(i+1 / 2)$ in $(36)$, we end up with the following decomposition for the matrix $H$

$$
\begin{aligned}
H \approx 3 & \left(\begin{array}{l}
\beta \\
2
\end{array}\right)\left[\left(\frac{2}{3}\right)^{2-\beta},\left(\frac{2}{5}\right)^{2-\beta}, \ldots,\left(\frac{2}{2 n-3}\right)^{2-\beta}\right]^{T} \\
& {\left[2^{-m(2-\beta)}, 2^{(1-m)(2-\beta)}, \cdots, 2^{-(2-\beta)}\right] } \\
+ & 21\left(\begin{array}{c}
\beta \\
3
\end{array}\right)\left[\left(\frac{2}{3}\right)^{3-\beta},\left(\frac{2}{5}\right)^{3-\beta}, \ldots,\left(\frac{2}{2 n-3}\right)^{3-\beta}\right]^{T} \\
& {\left[2^{-m(3-\beta)}, 2^{(1-m)(3-\beta)}, \cdots, 2^{-(3-\beta)}\right] } \\
- & 105\left(\begin{array}{c}
\beta \\
4
\end{array}\right)\left[\left(\frac{2}{3}\right)^{4-\beta},\left(\frac{2}{5}\right)^{4-\beta}, \ldots,\left(\frac{2}{2 n-3}\right)^{4-\beta}\right]^{T} \\
& {\left[2^{-m(4-\beta)}, 2^{(1-m)(4-\beta)}, \cdots, 2^{-(4-\beta)}\right] . }
\end{aligned}
$$

We observe from the decomposition (30) that the matrix $A_{r, l}$ can be approximately stored in $O(N)$ memories and the action of $A_{l, r}$ on any vector can be evaluated in $O(N)$ operations.

Remark 4.1. We conclude from Theorems 3 and 4 that the stiffness matrix A for the finite volume scheme (5), which is discretized on a locally refined composite mesh, can be stored in $O(N)$ of memories and $A w$ can be evaluated in $O(N \log N)$ operations for any vector $w \in \mathbb{R}^{N}$. 
Remark 4.2. We note from the expansion (33) for the $d_{i, j}$ that the local truncation error is not necessarily very small for $j \approx 1$. To retain the accuracy of the decompositions (34) and (35) for $D$ and $E$, we may restrict the range of $j$ for which we apply the efficient storage and fast matrix-vector mechanism in Theorem 4. For instance, we may use these decompositions for $\log N \leq j \leq N$ and just use the straightforward storage and matrixvector multiplication for the remaining $1 \leq j \leq \log N$. The incurred memory requirement and computational cost are $O(m \log N)$. Hence, as long as we choose $m$ to be bounded from above by $O(N / \log N)$ asymptotically, the memory requirement of the method is still $O(N)$ and the matrix-vector multiplication is still $O(N \log N)$.

We can similarly restrict the range of the index $i$ in the matrix decomposition of $G$ and $H$ and skip the details here.

\section{An efficient preconditioner}

The fast Krylov subspace method developed in section 4 reduces the computational cost of a conventional Krylov subspace method from $O\left(N^{2}\right)$ to $O(N \log N)$ per iteration. However, as the stiffness matrix $A$ is ill-conditioned, the number of iterations may still be large that in turn increases the overall computational cost. In this section we present an efficient preconditioner for the fast method.

Many circulant preconditioners have been developed for solving Toeplitz systems $[3,4]$. We present a preconditioner based on T. Chan's circulant preconditioner [4], which is outlined below. The Chan preconditioner $C_{F}=$ $C_{F}\left(T_{n}\right)$ is defined to be the minimizer of $\left\|T_{n}-C_{n}\right\|_{F}$ over all circulant matrices $C_{n}$. For a Toeplitz matrix $T_{n}$ of the form (32), the diagonals $c_{j}$ of $C_{F}\left(T_{n}\right)$ are given by

$$
c_{j}= \begin{cases}\frac{(n-j) t_{j}+j t_{j-n}}{n}, & 0 \leq j \leq n-1, \\ c_{n+j}, & 0<-j \leq n-1 .\end{cases}
$$

We note that the stiffness matrix $A$, which is of the form (7), is not Toeplitz. To motivate the development of a preconditioner based on T. Chen's preconditioner, we consider a special case of $K_{+}(x)=K_{-}(x)=K(x)$ and $\gamma=0.5$. Then we can construct an $m$-by- $m$ T Chan's circulant preconditioner $M_{1}$ for $A_{l, l}$ and an $(n-2) \times(n-2)$ T Chan's circulant precondtioner 
Table 1: Numerical results for the example in $\S 6.1$

\begin{tabular}{|r|c|c|c|}
\hline $\mathrm{n}$ & $\left\|u_{n}-u\right\|$ & $\left\|u_{\log n}-u\right\|$ & $\left\|u_{\sqrt{n}}-u\right\|$ \\
\hline 128 & $4.3546 \times 10^{-1}$ & $2.6805 \times 10^{-1}, m=7$ & $2.0315 \times 10^{-1}, m=11$ \\
256 & $4.0630 \times 10^{-1}$ & $2.3336 \times 10^{-1}, m=8$ & $1.3403 \times 10^{-1}, m=16$ \\
512 & $3.7909 \times 10^{-1}$ & $2.0315 \times 10^{-1}, m=9$ & $8.2504 \times 10^{-2}, m=22$ \\
1024 & $3.5370 \times 10^{-1}$ & $1.7685 \times 10^{-1}, m=10$ & $3.8488 \times 10^{-2}, m=32$ \\
8192 & $2.8730 \times 10^{-1}$ & $1.6668 \times 10^{-1}, m=13$ & $\mathrm{~N} / \mathrm{A}$ \\
\hline
\end{tabular}

$M_{2}$ for $A_{r, r}$. Then we define a block-diagonal-circulant-block preconditioner $M$ for $A$ in (7) as follows

$$
M:=\left[\begin{array}{cc}
\hat{M}_{1} & 0 \\
0 & M_{2}
\end{array}\right]
$$

To handle the first row and the first column of the matrix $A$ in (7), we extend the circulant matrix $M_{1}$ of order $m$ by one more row and one more column to a circulant matrix of order $m+1$.

\section{Numerical experiments}

We perform numerical experiments to investigate the performance of the fast finite volume method (5). In the numerical comparisons we measure the discrete maximum norm of the global truncation error

$$
\left\|u_{h}-u\right\|=\max _{0 \leq i \leq N}\left|u_{i}-u\left(x_{i}\right)\right| .
$$

\subsection{A one-sided space-fractional differential equation}

We consider the numerical simulation of the one-sided constant-coefficient problem (2) with $\beta=0.9$, which was introduced in section 1 and corresponds to problem (4) with $K=1, f=0, \gamma=1, \beta=0.9, u_{l}=0$, and $u_{r}=1$. The true solution $u$ is given in (1).

We take the advantage of the simplicity of the problem to investigate refining mechanisms on the boundary layer by comparing the performance of the corresponding finite volume method against the one on a uniform mesh. In the numerical experiments, we choose $m=\log n$ and $m=\lfloor\sqrt{n}\rfloor$ and denote the corresponding numerical solution as $u_{\log n}$ and $u_{\sqrt{n}}$, respectively. 

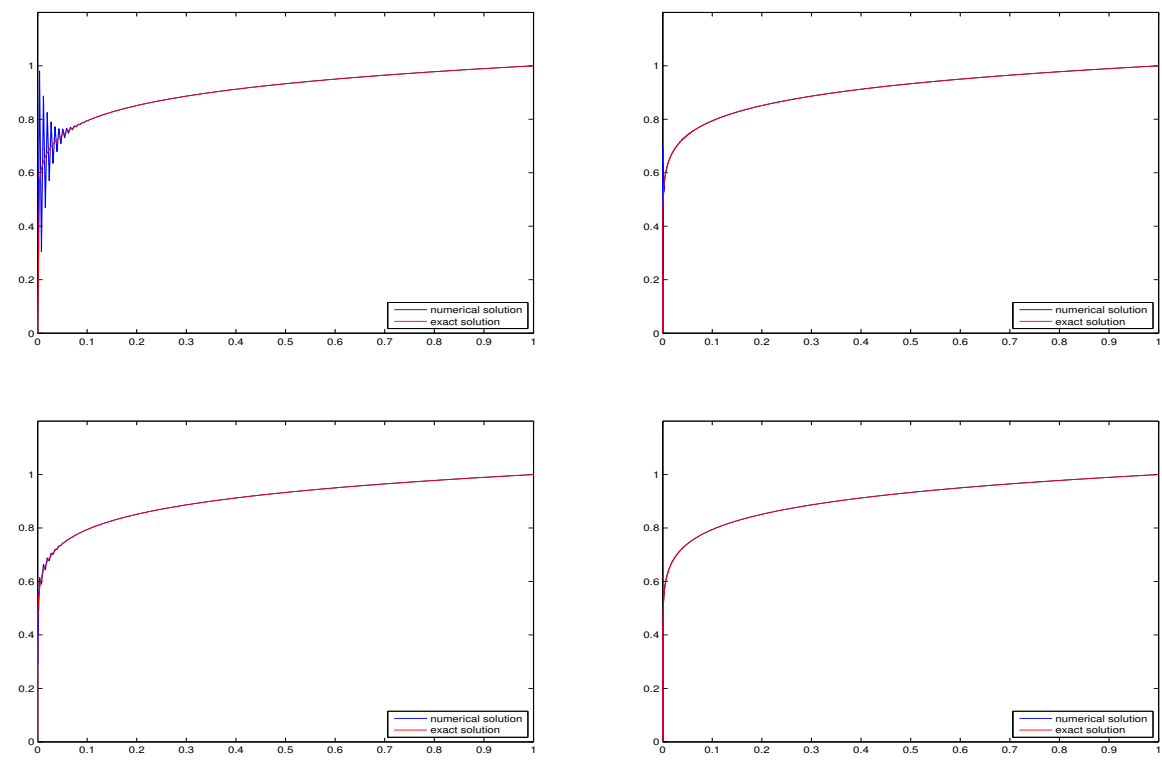

Figure 1: The example in $\S 6.1$ : In the first row are the numerical solutions on a uniform mesh of $n=256$ (left); and of $n=8192$ (right). In the second row are the numerical solutions on a locally refined composite mesh with $n=256$ and $m=\log n=8$ (left) or $m=\sqrt{n}=16$ (right). 
We use $u_{n}$ to denote the numerical solution on the uniform mesh with $n$ uniform cells. We used Gaussian elimination to solve the discrete systems.

We present the numerical results in Table 1 and some representative plots in Figure 1. We observe that with a uniform mesh of $n=256$ the numerical solution exhibits obvious numerical oscillations. With a geometrically refined mesh of $m=\log n=8$, the numerical solution eliminates almost all the oscillations with barely detectable errors near the boundary layer. With a geometrically refined mesh of $m=\lfloor\sqrt{n}\rfloor=16$, the numerical solution is free of numerical oscillations. A much refined uniform mesh of $n=8192$ has to be used to generate a numerical solution of the same quality. The numerical results in Table 1 reveal a similar trend but demonstrate more details. In particular, as more points were used in the boundary layer cell, the corresponding numerical solutions get more accurate. However, $m$ cannot be chosen too big. For example, $m=90$ for $n=8192=2^{13}$. The smallest subcell has a size about $10^{-30}$ that is well below the machine zero. In short, as $n$ gets not so large $\log n$ and $\sqrt{n}$ really does not make that much difference. These results seem to suggest that the finer the subcell is the better the numerical approximation is, provided that the subcell size is not close to machine zero.

\subsection{A two-sided space-fractional differential equation}

In this subsection we consider the numerical simulation of the Dirichlet boundary-value problem of a two-sided fractional differential equation (4) with $\gamma=0.5, K=1, u_{l}=0$, and $u_{r}=1$, and a source term given by

$$
f(x)=\frac{(1-\gamma)(1-\beta)}{\Gamma(\beta) x(1-x)^{1-\beta}} .
$$

The true solution is given by (1). We choose $\beta=0.9$ and $\beta=0.95$, so the true solutions exhibit steep boundary layers near the left endpoint $x=0$.

In the numerical experiments we investigate the performance of the finite volume method (5) on the locally refined composite mesh against the one on a uniform mesh as we did in the example in $\$ 6.1$. We use the numerical solutions by Gaussian elimination as a benchmark. We then solve the finite volume schemes by the conjugate gradient squared (CGS) method, by the fast conjugate gradient squared (FCGS) method in which the matrix-vector multiplication is carried out via the mechanism in $\S 4$, by the preconditioned fast conjugate gradient squared method with the T. Chan's circulant-block preconditioner (PFCGS) as described in $§ 5$. 
Table 2: Numerical results on a uniform mesh for the example in $\S 6.2$ with $\beta=0.9$

\begin{tabular}{|c|c|c|c|c|}
\hline & $\mathrm{n}$ & Error & Iterations & CPUs \\
\hline \multirow{7}{*}{ Gauss } & $2^{8}$ & $1.3700 \times 10^{-1}$ & & $0.00 \mathrm{~s}$ \\
\hline & $2^{9}$ & $1.2798 \times 10^{-1}$ & & $0.00 \mathrm{~s}$ \\
\hline & $2^{10}$ & $1.1949 \times 10^{-1}$ & & $0.04 \mathrm{~s}$ \\
\hline & $2^{11}$ & $1.1152 \times 10^{-1}$ & & $0.18 \mathrm{~s}$ \\
\hline & $2^{12}$ & $1.0407 \times 10^{-1}$ & & $1.11 \mathrm{~s}$ \\
\hline & $2^{13}$ & $9.7104 \times 10^{-2}$ & & $8.62 \mathrm{~s}$ \\
\hline & $2^{14}$ & $9.0605 \times 10^{-2}$ & & 101s \\
\hline \multirow{7}{*}{ CGS } & $2^{8}$ & $1.3700 \times 10^{-1}$ & 53 & $0.00 \mathrm{~s}$ \\
\hline & $2^{9}$ & $1.2798 \times 10^{-1}$ & 77 & $0.05 \mathrm{~s}$ \\
\hline & $2^{10}$ & $1.1949 \times 10^{-1}$ & 112 & $0.24 \mathrm{~s}$ \\
\hline & $2^{11}$ & $1.1152 \times 10^{-1}$ & 164 & $1.40 \mathrm{~s}$ \\
\hline & $2^{12}$ & $1.0407 \times 10^{-1}$ & 238 & $8.26 \mathrm{~s}$ \\
\hline & $2^{13}$ & $9.7104 \times 10^{-2}$ & 348 & $49 \mathrm{~s}$ \\
\hline & $2^{14}$ & $9.0605 \times 10^{-2}$ & 507 & $249 \mathrm{~s}$ \\
\hline \multirow{7}{*}{ FCGS } & $2^{8}$ & $1.3700 \times 10^{-1}$ & 53 & $0.10 \mathrm{~s}$ \\
\hline & $2^{9}$ & $1.2798 \times 10^{-1}$ & 77 & $0.23 \mathrm{~s}$ \\
\hline & $2^{10}$ & $1.1949 \times 10^{-1}$ & 112 & $0.43 \mathrm{~s}$ \\
\hline & $2^{11}$ & $1.1152 \times 10^{-1}$ & 164 & $1.69 \mathrm{~s}$ \\
\hline & $2^{12}$ & $1.0407 \times 10^{-1}$ & 238 & $3.10 \mathrm{~s}$ \\
\hline & $2^{13}$ & $9.7104 \times 10^{-2}$ & 348 & $17 \mathrm{~s}$ \\
\hline & $2^{14}$ & $9.0605 \times 10^{-2}$ & 507 & $40 \mathrm{~s}$ \\
\hline \multirow{7}{*}{ PFCGS } & $2^{8}$ & $1.3700 \times 10^{-1}$ & 9 & $0.01 \mathrm{~s}$ \\
\hline & $2^{9}$ & $1.2798 \times 10^{-1}$ & 9 & $0.03 \mathrm{~s}$ \\
\hline & $2^{10}$ & $1.1949 \times 10^{-1}$ & 9 & $0.05 \mathrm{~s}$ \\
\hline & $2^{11}$ & $1.1152 \times 10^{-1}$ & 10 & $0.14 \mathrm{~s}$ \\
\hline & $2^{12}$ & $1.0407 \times 10^{-1}$ & 10 & $0.18 \mathrm{~s}$ \\
\hline & $2^{13}$ & $9.7104 \times 10^{-2}$ & 10 & $0.76 \mathrm{~s}$ \\
\hline & $2^{14}$ & $9.0605 \times 10^{-2}$ & 12 & $1.24 \mathrm{~s}$ \\
\hline
\end{tabular}


Table 3: Numerical results on a composite mesh for the example in $\S 6.2$ with $\beta=0.9$

\begin{tabular}{|c|cc|c|c|}
\hline & $\mathrm{m}$ & $\mathrm{n}$ & $\left\|u_{h}-u\right\|$ & \# of iterations \\
\hline \multirow{3}{*}{ Gauss } & $2^{3}$ & $2^{8}$ & $7.9306 \times 10^{-2}$ & \\
& $2^{4}$ & $2^{9}$ & $4.2326 \times 10^{-2}$ & \\
& $2^{5}$ & $2^{10}$ & $1.3025 \times 10^{-2}$ & \\
\hline \multirow{4}{*}{ CGS } & $2^{3}$ & $2^{8}$ & $7.9306 \times 10^{-2}$ & 58 \\
& $2^{4}$ & $2^{9}$ & $4.2326 \times 10^{-2}$ & 106 \\
& $2^{5}$ & $2^{10}$ & $1.3025 \times 10^{-2}$ & 256 \\
\hline \multirow{3}{*}{ FCGS } & $2^{3}$ & $2^{8}$ & $7.9306 \times 10^{-2}$ & 58 \\
& $2^{4}$ & $2^{9}$ & $4.2326 \times 10^{-2}$ & 106 \\
& $2^{5}$ & $2^{10}$ & $1.3025 \times 10^{-2}$ & 256 \\
\hline \multirow{3}{*}{ PFCGS } & $2^{3}$ & $2^{8}$ & $7.9306 \times 10^{-2}$ & 13 \\
& $2^{4}$ & $2^{9}$ & $4.2326 \times 10^{-2}$ & 16 \\
& $2^{5}$ & $2^{10}$ & $1.3025 \times 10^{-2}$ & 25 \\
\hline
\end{tabular}

We present the numerical results in Tables $2-5$. We did not present the CPU times for Tables 3 and 5, as the mesh sizes are coarse and the CPU time is not even accurately measurable to show any reasonable comparison. We reach the following observations from these tables: (i) The finite volume method on locally refined composite meshes generates performs much more effectively than the one on a uniform mesh, in approximating numerical solutions with boundary layers. For example, the finite volume method on a locally refined composite mesh with $n=256$ and $m=8$ generates more accurate numerical solutions than that on a uniform mesh of $n=16384$. (ii) All the iterative methods generate numerical solutions with the same accuracy as Gaussian elimination. (iii) The PFCGS method significantly reduce the number of iterations in the FCGS method, which only increases very slowly as the number of unknowns doubles.

\subsection{Impact of the matrix expansions}

The local truncation error in the expansion (33) for $d_{i, j}$ may become nonnegligible as the column index $j$ is close to 1 . Similarly, the local truncation error in the expansion (36) for $g_{i, j}$ may become non-negligible too, as the row index $i$ is close to 1 and the column index $j$ is close to $m$. As we discussed in $\S 5$, we could restrict the range of $i$ and $j$ in the fast matrix- 
Table 4: Numerical results on a uniform mesh for the example in $\S 6.2$ with $\beta=0.95$

\begin{tabular}{|c|c|c|c|c|}
\hline & $\mathrm{n}$ & Error & Iterations & CPUs \\
\hline \multirow{7}{*}{ Gauss } & $2^{8}$ & $1.8827 \times 10^{-1}$ & & $0.01 \mathrm{~s}$ \\
\hline & $2^{9}$ & $1.8206 \times 10^{-1}$ & & $0.01 \mathrm{~s}$ \\
\hline & $2^{10}$ & $1.7596 \times 10^{-1}$ & & $0.05 \mathrm{~s}$ \\
\hline & $2^{11}$ & $1.7002 \times 10^{-1}$ & & $0.25 \mathrm{~s}$ \\
\hline & $2^{12}$ & $1.6425 \times 10^{-1}$ & & $1.25 \mathrm{~s}$ \\
\hline & $2^{13}$ & $1.5867 \times 10^{-1}$ & & $9.76 \mathrm{~s}$ \\
\hline & $2^{14}$ & $1.5327 \times 10^{-1}$ & & $97 \mathrm{~s}$ \\
\hline \multirow{7}{*}{ CGS } & $2^{8}$ & $1.8827 \times 10^{-1}$ & 46 & $0.01 \mathrm{~s}$ \\
\hline & $2^{9}$ & $1.8206 \times 10^{-1}$ & 66 & $0.01 \mathrm{~s}$ \\
\hline & $2^{10}$ & $1.7596 \times 10^{-1}$ & 94 & $0.18 \mathrm{~s}$ \\
\hline & $2^{11}$ & $1.7002 \times 10^{-1}$ & 133 & $0.86 \mathrm{~s}$ \\
\hline & $2^{12}$ & $1.6425 \times 10^{-1}$ & 188 & $4.94 \mathrm{~s}$ \\
\hline & $2^{13}$ & $1.5867 \times 10^{-1}$ & 266 & $30.78 \mathrm{~s}$ \\
\hline & $2^{14}$ & $1.5327 \times 10^{-1}$ & 379 & $187 \mathrm{~s}$ \\
\hline \multirow{7}{*}{ FCGS } & $2^{8}$ & $1.8827 \times 10^{-1}$ & 46 & $0.05 \mathrm{~s}$ \\
\hline & $2^{9}$ & $1.8206 \times 10^{-1}$ & 66 & $0.16 \mathrm{~s}$ \\
\hline & $2^{10}$ & $1.7596 \times 10^{-1}$ & 94 & $0.29 \mathrm{~s}$ \\
\hline & $2^{11}$ & $1.7002 \times 10^{-1}$ & 133 & $1.16 \mathrm{~s}$ \\
\hline & $2^{12}$ & $1.6425 \times 10^{-1}$ & 188 & $2.00 \mathrm{~s}$ \\
\hline & $2^{13}$ & $1.5867 \times 10^{-1}$ & 266 & $12 \mathrm{~s}$ \\
\hline & $2^{14}$ & $1.5327 \times 10^{-1}$ & 379 & $27 \mathrm{~s}$ \\
\hline \multirow{7}{*}{ PFCGS } & $2^{8}$ & $1.8827 \times 10^{-1}$ & 8 & $0.02 \mathrm{~s}$ \\
\hline & $2^{9}$ & $1.8206 \times 10^{-1}$ & 8 & $0.02 \mathrm{~s}$ \\
\hline & $2^{10}$ & $1.7596 \times 10^{-1}$ & 9 & $0.05 \mathrm{~s}$ \\
\hline & $2^{11}$ & $1.7002 \times 10^{-1}$ & 10 & $0.09 \mathrm{~s}$ \\
\hline & $2^{12}$ & $1.6425 \times 10^{-1}$ & 10 & $0.14 \mathrm{~s}$ \\
\hline & $2^{13}$ & $1.5867 \times 10^{-1}$ & 10 & $0.66 \mathrm{~s}$ \\
\hline & $2^{14}$ & $1.5327 \times 10^{-1}$ & 11 & $1.00 \mathrm{~s}$ \\
\hline
\end{tabular}


Table 5: Numerical results on a composite mesh for the example in $\S 6.2$ with $\beta=0.95$

\begin{tabular}{|ccccc|}
\hline & $\mathrm{m}$ & $\mathrm{n}$ & Error & Iterations \\
\hline \multirow{3}{*}{ Gauss } & $2^{3}$ & $2^{8}$ & $1.4379 \times 10^{-1}$ & \\
& $2^{4}$ & $2^{9}$ & $1.0491 \times 10^{-1}$ & \\
& $2^{5}$ & $2^{10}$ & $5.8194 \times 10^{-2}$ & \\
\hline \multirow{4}{*}{ CGS } & $2^{3}$ & $2^{8}$ & $1.4379 \times 10^{-2}$ & 48 \\
& $2^{4}$ & $2^{9}$ & $1.0491 \times 10^{-1}$ & 77 \\
& $2^{5}$ & $2^{10}$ & $5.8194 \times 10^{-2}$ & 142 \\
\hline \multirow{3}{*}{ FCGS } & $2^{3}$ & $2^{8}$ & $1.4379 \times 10^{-1}$ & 48 \\
& $2^{4}$ & $2^{9}$ & $1.0491 \times 10^{-1}$ & 78 \\
& $2^{5}$ & $2^{10}$ & $5.8194 \times 10^{-2}$ & 150 \\
\hline \multirow{3}{*}{ PFCGS } & $2^{3}$ & $2^{8}$ & $1.4379 \times 10^{-1}$ & 9 \\
& $2^{4}$ & $2^{9}$ & $1.0491 \times 10^{-1}$ & 13 \\
& $2^{5}$ & $2^{10}$ & $5.8194 \times 10^{-2}$ & 16 \\
\hline
\end{tabular}

vector multiplication and leave the remaining (relatively small) number of rows and columns computed using the conventional way.

In this subsection we look at the impact of restricting the index $i$ and/or $j$ in these fast matrix-vector multiplications.

Scenario 1:. When we use the matrix-vector multiplication $D w_{1}$ and $E w_{1}$ for any vector $w_{1}$, we restrict the row index $i$ to the range of $1 \leq i \leq m-\log m$. Similarly, when we evaluate $G w_{2}$ and $H w_{2}$, we restrict the column index $j$ to the range of $1 \leq j \leq m-\log m$. This was used in the numerical example runs in $§ 6.2$.

Scenario 2: When we use the matrix-vector multiplication $D w_{1}$ and $E w_{1}$ for any vector $w_{1}$, we restrict the column index $j$ to the range of $\log m+1 \leq$ $j \leq n-2$. Similarly, when we evaluate $G w_{2}$ and $H w_{2}$, we restrict the row index $i$ to the range of $\log m+1 \leq i \leq n-2$. In both Scenarios 1 and 2, the discrepancy of the expressions due to different expressions for $d_{(m, 1)}$ and $g_{(1, m)}$ is avoided automatically.

Scenario 3: We impose both constraints in Scenarios 1 and 2.

Scenario $4:$ In this scenario, we do not use the expansions for $D, E, G$, and $H$. Instead, we use the original matrix $A_{l, r}$ and $A_{r, l}$. 
Table 6: Numerical results for the example in $\S 6.3$ with $\beta=0.9$

\begin{tabular}{|c|cc|c|}
\hline$i, j$ & $\mathrm{~m}$ & $\mathrm{n}$ & $\left\|u_{h}-u\right\|$ \\
\hline \multirow{3}{*}{ Scenario 1 } & $2^{3}$ & $2^{8}$ & $7.9306 \times 10^{-2}$ \\
& $2^{4}$ & $2^{9}$ & $4.2326 \times 10^{-2}$ \\
& $2^{5}$ & $2^{10}$ & $1.3025 \times 10^{-2}$ \\
\hline \multirow{3}{*}{ Scenario 2 } & $2^{3}$ & $2^{8}$ & $7.8984 \times 10^{-2}$ \\
& $2^{4}$ & $2^{9}$ & $4.2121 \times 10^{-2}$ \\
& $2^{5}$ & $2^{10}$ & $1.2961 \times 10^{-2}$ \\
\hline & $2^{3}$ & $2^{8}$ & $7.8608 \times 10^{-2}$ \\
Scenario 3 & $2^{4}$ & $2^{9}$ & $4.2111 \times 10^{-2}$ \\
& $2^{5}$ & $2^{10}$ & $1.2961 \times 10^{-2}$ \\
\hline \multirow{3}{*}{ Scenario 4 } & $2^{3}$ & $2^{8}$ & $7.8590 \times 10^{-2}$ \\
& $2^{4}$ & $2^{9}$ & $4.2110 \times 10^{-2}$ \\
& $2^{5}$ & $1.2961 \times 10^{-2}$ \\
\hline
\end{tabular}

As we only investigate the accuracy of the expansions, we use Gaussian elimination in the computations in this example. We present the numerical results in Table 6 and observe the impact of the restrictions, which is deemed to be not significant and are very close to the solutions without any matrix compressions.

We end this section by briefly commenting on the issue of convergence rate of the finite volume scheme. Previous studies showed that finite volume methods are anticipated to have a second-order convergence rate with respect to the mesh size on a uniform mesh [9, 13, 20, 27, 28] without using a Richardson extrapolation. This is in contrast to the Meerschaert-Tadjeran finite difference scheme, in which a Richardson extrapolation is required to recover a second-order accuracy [18].

The objectives of this paper are as follows: (i) to develop a finite volume scheme on a locally refined, geometrically gridded mesh to effectively resolve the boundary layer present in the solutions to space-fractional differential equations; and (ii) to explore the structure of the scheme to efficiently solve the resulting discrete algebraic system. The numerical results in this section demonstrate that the fast finite volume scheme fulfills these goals.

This paper represents only a preliminary step in the full resolution of the problem. In this paper we measure the discrete maximum error (41) to 
make sure that a uniform convergence is achieved. However, the issue of convergence rate of the finite volume scheme remains to be analyzed: (i) an appropriate measurement of the truncation error, that is, what is a proper more to measure the error which is actually a tricky issue in the context of second-order advection-diffusion equations [8, 17, 22]. (ii) The error is measured with respect what parameter. Apparently, the mesh size on the uniform partition is not proper. An often used parameter in an adaptive refinement is the total number of nodes. This again relies on the local refinement strategy. (iii) The convergence rate also depends the regularity of the true solution. As the solution has a boundary layer and a low regularity, the exact convergence rate of the finite volume scheme remains to be fully investigated. All these issues will be studied in the near future.

\section{Extensions}

In this section we outline the development of the fast finite volume method to problem (2) in which boundary layers appear at both endpoints $x=0$ and $x=1$. Similarly to what we did in $\S 2$, we first introduce a uniform mesh $h=1 / n$. Then we introduce geometrically refined meshes within the boundary layer cell $[0, h]$ and $[1-h, 1]$. For simplicity of presentation, we assume that the number of locally refined mesh points are $m$ in both cells. In this way, the grid points are given by $x_{0}, x_{1}, \cdots, x_{2 m+n}$. The corresponding stiffness matrix $A$ can be expressed in the following block form

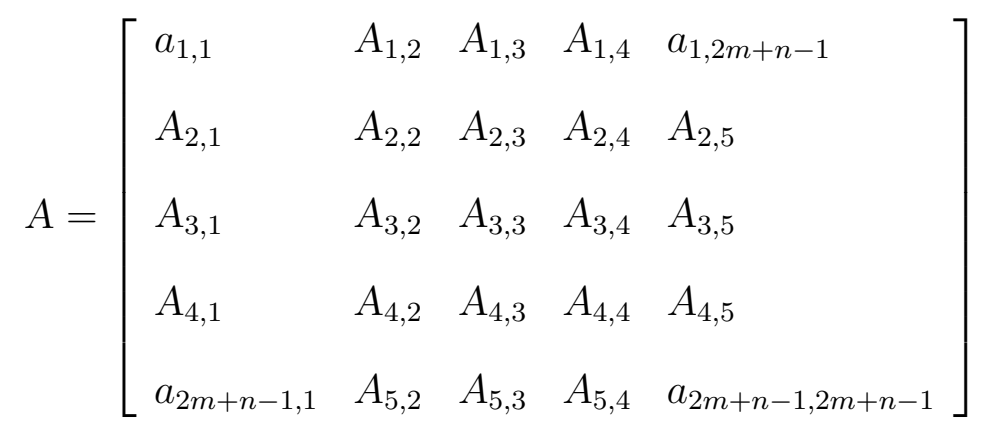

The stiffness matrix for the case of only one boundary layer appears at the left endpoint corresponds to a matrix with only the three-by-three block matrix given in (7), to which the matrix decompositions were derived in $\S 4$ and $\S 5$. By symmetry, the three-by-three block matrix consisting of $A_{3,3}$, $A_{3,4}, A_{3,5}, A_{4,3}, A_{4,4}, A_{4,5}, A_{5,3}, A_{5,4}$, and $a_{2 m+n-1,2 m+n-1}$ can be expanded 
in a similar fashion. We also notice that $A_{1,4}$ and $A_{2,5}$ are row vectors while $A_{4,1}$ and $A_{5,2}$ are column vectors.

Hence we need only to study the matrices $A_{2,4}$ and $A_{4,2}$. By symmetry, we focus only on the matrix $A_{4,2}$. A straightforward algebra yields

$$
\begin{aligned}
A_{4,2 ; i, j}= & \frac{\gamma K_{j-\frac{1}{2}}}{\Gamma(\beta+1) h_{i}^{1-\beta}} f_{i, j-1}-\frac{\gamma K_{i+\frac{1}{2}}}{\Gamma(\beta+1) h_{j}^{1-\beta}} f_{i, j}, \\
& m+n \leq i \leq 2 m+n-1, \quad 2 \leq j \leq m-3
\end{aligned}
$$

for $m+n \leq i \leq 2 m+n-1$ and $2 \leq j \leq m-3$. Here $f_{i, j}$ are defined by

$$
\begin{gathered}
f_{i, j}=\left(2^{m+1-j}-3 \cdot 2^{(2 m+n)-(i+j)-1}-1\right)^{\beta} \\
-3\left(2^{m+1-j}-3 \cdot 2^{(2 m+n)-(i+j)-1}-2\right)^{\beta} \\
\quad+2\left(2^{m+1-j}-3 \cdot 2^{(2 m+n)-(i+j)-1}-4\right)^{\beta} \cdot \\
\left(2^{m+1-j}-3 \cdot 2^{(2 m+n)-(i+j)-1}-1\right)^{\beta} \\
=\left(2^{m+1-j}\right)^{\beta}\left(1-\frac{3 \cdot 2^{(2 m+n)-(i+j)-1}}{2^{m+1-j}}-\frac{1}{2^{m+1-j}}\right)^{\beta} \\
=\left(2^{m+1-j}\right)^{\beta}\left(1-3 \cdot 2^{m+n-i-2}-2^{j-m-1}\right)^{\beta}
\end{gathered}
$$

We can similarly expand the right-hand side by a Taylor expansion. After some cancellations, $f_{i, j}$ can be expressed in similar forms to those in $\S 5$. A similar derivation to $\S 5$ leads to an effective storage and a fast matrix-vector multiplication mechanism.

\section{Acknowledgements}

This work was supported in part by the National Science Foundation under Grants EAR-0934747 and DMS-1216923, by the National Natural Science Foundation of China under Grants 91130010 and 11471194, and by the State Scholarship Fund from China Scholarship Council under Grant 201306220110. The authors would like to express their sincere thanks to the referees for their very helpful comments and suggestions, which greatly improved the quality of this paper. 
[1] R.A. Adams and J.J.F. Fournier, Sobolev Spaces, Elsevier, San Diego, 2003.

[2] D. Benson, S.W. Wheatcraft, and M.M. Meerschaert, The fractionalorder governing equation of Lévy motion, Water Resour. Res., 36 (2000), $1413-1423$.

[3] R.H. Chan and M.K. Ng, Conjugate Gradient Methods for Toeplitz Systems, SIAM Review, 38 (1996), 427-482.

[4] T. Chan, An optimal circulant preconditioner for Toeplitz systems, SIAM J. Sci. Stat. Comput., 9 (1988), 766-771.

[5] S. Chen, F. Liu, X. Jiang, I. Turner and V. Anh, A fast semi-implicit difference method for a nonlinear two-sided space-fractional diffusion equation with variable diffusivity coefficients, Appl. Math. Comp., 257 (2015), 591-601.

[6] D. del-Castillo-Negrete, B.A. Carreras, and V. E. Lynch, Fractional diffusion in plasma turbulence, Phys. Plasmas, 11 (2004), 3854.

[7] V.J. Ervin and J.P. Roop, Variational formulation for the stationary fractional advection dispersion equation, Numer. Methods. Partial Differential Eq, 22 (2005), 558-576.

[8] P.A. Farrell, A.F. Hegarty, J.J.H. Miller, E. O'Riordan, and G.I. Shishkin, Robust Computational Techniques for Boundary Layers, Applied Mathematics 16, Chapman \& Hall/CRC, Boca Raton, Florida, 2000 .

[9] L.B. Feng, P. Zhuang, F. Liu, I. Turner, Stability and convergence of a new finite volume method for a two-sided space-fractional diffusion equation, Appl. Math. Comp., in press, 10.1016/j.amc.2014.12.060.

[10] H. Hejazi, T. Moroney and F. Liu, Stability and convergence of a finite volume method for the space fractional advection-dispersion equation, J. Comput. Appl. Math., 255 (2014)684-697.

[11] B. Jin, R. Lazarov, and J. Pasciak, Variational formulation of problems involving fractional order differential operators, arXiv:1307.4795 [math.NA] 
[12] F. Liu, V. Anh, and I. Turner, Numerical solution of the space fractional Fokker-Planck equation, J. Comput. Appl. Math. 166 (2004), pp. 209219.

[13] F. Liu, P. Zhuang, I. Turner, K. Burrage and V. Anh, A new fractional finite volume method for solving the fractional diffusion equation, Appl. Math. Modelling, 38 (2014), 3871-3878.

[14] M.M. Meerschaert and C. Tadjeran, Finite difference approximations for fractional advection-dispersion flow equations, J. Comput. Appl. Math., 172 (2004) 65-77.

[15] R. Metzler and J. Klafter, The random walk's guide to anomalous diffusion: A fractional dynamics approach, Phys. Rep., 339 (2000), 1-77.

[16] I. Podlubny, Fractional Differential Equations, Academic Press, New York, 1999.

[17] H.-G. Roos, M. Stynes, and L. Tobiska, Robust Numerical Methods for Singularly Perturbed Differential Equations: Convection-DiffusionReaction and Flow Problems, Springer-Verlag, Berlin, 2008.

[18] C. Tadjeran, M.M. Meerschaert, and H.P. Scheffler, A second-order accurate numerical approximation for the fractional diffusion equation, $J$. Comput. Phys., 213 (2006), 205-213.

[19] H. Wang and T.S. Basu, A fast finite difference method for twodimensional space-fractional diffusion equations, SIAM J. Sci. Comput., 34 (2012), A2444-A2458.

[20] H. Wang and N. Du, A superfast-preconditioned iterative method for steady-state space-fractional diffusion equations, J. Comput. Phys., 240 (2013), 49-57.

[21] H. Wang and N. Du, Fast alternating-direction finite difference methods for three-dimensional space-fractional diffusion equations, J. Comput. Phys., 258 (2013), 305-318.

[22] H. Wang and K. Wang, Uniform estimates for Eulerian-Lagrangian methods for singularly perturbed time-dependent problems, SIAM J. Numer. Anal., 45 (2007), 1305-1329. 
[23] H. Wang, K. Wang, and T. Sircar, A direct $O\left(N \log ^{2} N\right)$ finite difference method for fractional diffusion equations, J. Comput. Phys., 229 (2010), 8095-8104.

[24] H. Wang, D. Yang, S. Zhu, Inhomogeneous Dirichlet boundary-value problems of space-fractional diffusion equations and their finite element approximations, SIAM J. Numer. Anal., 52 (2014), 1292-1310.

[25] H. Wang and X. Zhang, A high-accuracy preserving spectral Galerkin method for the Dirichlet boundary-value problem of variable-coefficient conservative fractional diffusion equations, J. Comput. Phys., 281 (2015), 67-81.

[26] Q. Yang, I. Turner, F. Liu and M. Ilis, Novel numerical methods for solving the time-space fractional diffusion equation in 2D, SIAM J. Sci. Comput., 33 ( 2011) 1159-1180.

[27] Q. Yang, I. Turner, T. Moroney and F. Liu, A finite volume scheme with preconditioned Lanczos method for two-dimensional space-fractional reaction-diffusion equations, Appl. Math. Modelling, 38 (2014), 37553762 .

[28] P. Zhuang, F. Liu, I. Turner and Y.T. Gu, Finite volume and finite element methods for solving a one-dimensional space-fractional Boussinesq equation, Appl. Math. Modelling, 38 (2014), 3860-3870. 